\title{
Aharonov-Bohm effect of excitons in nano-rings
}

\author{
Hui $\mathrm{Hu}^{1}$, Jia-Lin Zhu ${ }^{1,2}$, Dai-Jun $\mathrm{Li}^{1}$ and Jia-Jiong Xiong ${ }^{1}$ \\ ${ }^{1}$ Department of Physics, Tsinghua University, Beijing 100084, P. R. China \\ ${ }^{2}$ Center for Advanced Study, Tsinghua University, Beijing 100084, P. R. China
}

(October 27, 2018)

\begin{abstract}
The magnetic field effects on excitons in an InAs nano-ring are studied theoretically. By numerically diagonalizing the effective-mass Hamiltonian of the problem, which can be separated into terms in centre-of-mass and relative coordinates, we calculate the low-lying exciton energy levels and oscillator strengths as a function of the width of the ring and the strength of the external magnetic field. The analytical results are obtained for a narrow-width nano-ring in which the radial motion is the fastest one and adiabatically decoupled from the azimuthal motions. It is shown that in the presence of Coulomb correlation, the so called Aharonov-Bohm effect of excitons exists in a finite (but small) width nano-ring. However, when the ring width becomes large, the non-simply-connected geometry of nano-rings is destroyed and in turn yields the suppression of Aharonov-Bohm effect. The conditional probability distribution calculated for the low-lying exction states allows identification of the presence of Aharonov-Bohm effect. The linear optical susceptibility is also calculated as a function of the magnetic field, to be confronted with the future measurements of optical emission experiments on InAs nano-rings.

PACS numberes: 73.20.Dx, 71.35.-y, 03.65.Bz, 78.66.Fd
\end{abstract}

\section{INTRODUCTION}

Recently, Lorke and collaborators demonstrated the realization of self-assembled InAf nano-rings inside a completed field-effect transistorl 3 . Such small nano-rings (with typical inner/outer radius of 20/100 $\mathrm{nm}$ and $2-3$ $\mathrm{nm}$ in height) allow one to study the new non-simplyconnected geometry where electrons or holes could propagate coherently (non-diffusively) all throughout 20 . In particular, they offer a unique opportunity to explore the so called "Aharonov-Bohm effect" (ABE) of an exciton, an interesting concept suggested by Chaplik, Römer and Raikh21.22.

Chaplik first predicted the Aharonov-Bohm (AB) oscillation of excitpnic levels in one-dimensional quantum ring structures 21 , and most recently, Römer and Raikh found the similar results with a short ranged interaction potential by using a quite different analytical approach 22 . In contrast to the general belief that an exciton, being a bound state of electron and hole and thus a neutral entity, should not be sensitive to the applied flux, they revealed the possibility of a non-vanishing $\mathrm{ABE}$ in one dimensional rings caused by the finite confinement of an exciton. On the other hand, up to now the influence of the ring width on $\mathrm{ABE}$ has rarely been investigated. Only recently did Song and Ulloa study the magnetic field effect on excitons in a finite width nano-ring. They found that the excitons in nano-rings behave to a great extent as those in quantum dots of similar dimensions and the finite width of the nano-rings can suppress com pletely the $\mathrm{ABE}$ predicted for one-dimensional ring 23 . At the moment, however, to what extent does the $\mathrm{ABE}$ exist in the quasi-one-dimensional (or more important, the realistic) nano-rings is still unclear and is an open subject for research.

In this paper, we would like to investigate systematically the magnetic field effect on an exciton within a simplified model Hamiltonian, which is applicable to the reglistic self-assembled semiconducting InAs nanorings 3 [24. By diagonalizing the effective-mass Hamiltonian of the problem and calculating the low-lying exciton energy levels and oscillator strengths as a function of the width of the ring and the strength of the external magnetic field, we show an evident ABE for nano-rings with a finite but small ring width. The numerical results are well interpreted with the analytical results for a narrow-width nano-ring in which the radial motion is the fastest one and adiabatically decoupled from the azimuthal motions, and also well understood by the conditional probability distribution calculated for the low-lying exciton states. The following is a summary of our main results:

(i) In the presence of Coulomb interaction between electrons and holes, there is no ABE for the ground excitonic state due to the intrinsic divergence of ground state energy of one-dimensional excitons.

(ii) Some of the low-lying excited energy levels and oscillator strengths of the exciton show a periodic, AharonovBohm-type oscillation, as a function of the magnetic field. The periodicity of oscillations is equal to $\Phi_{0} \equiv \frac{h}{e}$ - the universal flux quantum.

(iii) In addition to the overall blue shifts caused by the diamagnetic effect, the linear optical susceptibility traces apparently show ABE oscillations with the magnetic field for some of excited states.

The remainder of this paper is organized as follows. In Sec. II, we present the simple model for the system and solution method. As a first approximation, the nano-ring structure is modeled by a double-well like confinement potential in which we can separate the Hamiltonian into terms in centre-of-mass and relative coordinates. Subsequently (Sec. III), we give the analytical results for the narrow-width nano-rings with $m_{e}^{*}=m_{h}^{*}$. By calculating the tunneling possibility for three typical electron-hole interaction potential $V_{e-h}(\varphi)$, we explore the underlying 
physics of ABE, and the main results of Chaplik, Römer and Raikh are recovered. Sec. IV is devoted to the detailed discussion of numerical results for different ring widths and magnetic fields, including the low-lying energy levels, oscillator strengths, conditional probability distributions, pair-correlation functions, and the linear optical susceptibility. Finally, we summarize our results in Sec. V.

\section{MODEL AND NUMERICAL METHOD}

We start from a simplified model Hamiltonian for a two-dimensional exciton in an InAs nano-ring and in a static magnetic field simulating recent experimental nano-ring structures 324 . The exciton is described by an electron-hole pair $(i=e, h)$ with an effective band edge mass $m_{i}^{*}$ moving in the $\mathrm{x}-\mathrm{y}$ plane. The ring-like structure is well described by a double-well potential, $U\left(\overrightarrow{\mathbf{r}}_{i}\right)=\frac{1}{2 R_{0}^{2}} m_{i}^{*} \omega_{i}^{2}\left(\overrightarrow{\mathbf{r}}_{i}^{2}-R_{0}^{2}\right)^{2}$, which reproduces a soft barrier $\frac{m_{i}^{*} \omega_{i}^{2} R_{0}^{2}}{2}$ at the center of the sample produced by self-assembly 1 目, 4 . Here, $R_{0}$ is the radius of the ring and $\omega_{i}$ is the characteristic frequency of the radial confinement, giving a characteristic ring width $W \approx 2 \sqrt{\frac{\hbar}{2 m_{i}^{*} \omega_{i}}}$ for each particle. The whole system is subjected to an external magnetic field perpendicular to the $\mathrm{x}-\mathrm{y}$ plane. The resulting model Hamiltonian is thus given by:

$\mathcal{H}=\sum_{i=e, h}\left[\frac{\left(\overrightarrow{\mathbf{p}}_{i}+q_{i} \overrightarrow{\mathbf{A}}\left(\overrightarrow{\mathbf{r}}_{i}\right)\right)^{2}}{2 m_{i}^{*}}+U\left(\overrightarrow{\mathbf{r}}_{i}\right)\right]-\frac{e^{2}}{4 \pi \varepsilon_{0} \varepsilon_{r}\left|\overrightarrow{\mathbf{r}}_{e}-\overrightarrow{\mathbf{r}}_{h}\right|}$,

where $\overrightarrow{\mathbf{r}}_{i}=\left(x_{i}, y_{i}\right)$ and $\overrightarrow{\mathbf{p}}_{i}=-i \hbar \vec{\nabla}_{i}$ denote the position vector and momentum operator, $\varepsilon_{0}$ is the vacuum permittivity, and $\varepsilon_{r}$ is the static dielectric constant of the host semiconductor. $q_{e}=-e$ and $q_{h}=+e$. We use symmetric gauge to introduce the external magnetic field, i.e., $\overrightarrow{\mathbf{A}}\left(\overrightarrow{\mathbf{r}}_{i}\right)=\frac{1}{2} \overrightarrow{\mathbf{B}} \times \overrightarrow{\mathbf{r}}_{i}$.

It should be pointed out that the present double-well like confinement potential can be rewritten as $U\left(\overrightarrow{\mathbf{r}}_{i}\right)=$ $\frac{1}{2} m_{i}^{*} \omega_{i}^{2}\left(r_{i}-R_{0}\right)^{2} \frac{\left(r_{i}+R_{0}\right)^{2}}{R_{0}^{2}}$. If one replaces the operator $r_{i}$ in factor $\frac{\left(r_{i}+R_{0}\right)^{2}}{R_{0}^{2}}$ by its mean value $\left\langle r_{i}\right\rangle=R_{0}$, the confinement motential returns to the widely used parabolic

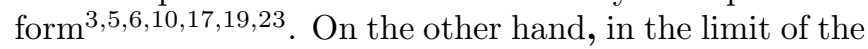
small radius $R_{0}$ or for a small potential strength $\omega_{i}$, the soft barrier at the ring center is very weak, and the description of the nano-ring is more close to that of a quantum dot. For a fixed ring width (or potential strength), the crossover from nano-rings to quantum dots is determined by $R_{0} \sim \frac{\sqrt{2}}{2} W$ or $\frac{\hbar \omega_{i}}{2} \sim \frac{m_{i}^{*} \omega_{i}^{2} R_{0}^{2}}{2}$, which means the lowest energy of radial confinement is comparable to the soft barrier at the ring center. It should also be pointed out that our double-well like confinement potential has been used to calculate the far-infrared spectroscopy for a two-electron nano-ringes, in good agreement with the recent experiment done by Lorke et al. 3 . In Fig. 1, we show the schematic geometry of InAs nano-rings with radius $R_{0}=20 \mathrm{~nm}$ for electrons. (a) and (b) correspond to two different ring widths $W=10$ and $7 \mathrm{~nm}$, respectively.

In terms of the relative coordinate $\overrightarrow{\mathbf{r}}=\overrightarrow{\mathbf{r}}_{e}-\overrightarrow{\mathbf{r}}_{h}$ and center-of-mass coordinate $\overrightarrow{\mathbf{R}}=\frac{m_{e}^{*} \overrightarrow{\mathbf{r}}_{e}+m_{h}^{*} \overrightarrow{\mathbf{r}}_{h}}{m_{e}^{*}+m_{h}^{*}}$ the model Hamiltonian can be separated into the motion of center of mass, relative motion of the electron-hole pair, and the mixed part:

$$
\begin{aligned}
\mathcal{H}= & \mathcal{H}_{c m}(\overrightarrow{\mathbf{R}})+\mathcal{H}_{r e l}(\overrightarrow{\mathbf{r}})+\mathcal{H}_{m i x}(\overrightarrow{\mathbf{R}}, \overrightarrow{\mathbf{r}}) \\
\mathcal{H}_{c m}(\overrightarrow{\mathbf{R}})= & \frac{\overrightarrow{\mathbf{P}}_{c m}^{2}}{2 M}+\frac{1}{2} M \omega_{c m}^{2} \frac{\left(\overrightarrow{\mathbf{R}}^{2}-R_{0}^{2}\right)^{2}}{R_{0}^{2}} \\
\mathcal{H}_{r e l}(\overrightarrow{\mathbf{r}})= & \frac{\overrightarrow{\mathbf{p}}_{r e l}^{2}}{2 \mu}+\frac{e^{2} B^{2}}{8 \mu} r^{2} \\
& +\frac{e B}{2}\left(\frac{1}{m_{e}^{*}}-\frac{1}{m_{h}^{*}}\right) \overrightarrow{\mathbf{r}} \times \overrightarrow{\mathbf{p}}_{r e l} \\
& +\frac{\mu}{2} \frac{\left(m_{h}^{* 3} \omega_{e}^{2}+m_{e}^{* 3} \omega_{h}^{2}\right)}{M^{3}} \frac{r^{4}}{R_{0}^{2}} \\
& -\mu \omega_{r e l}^{2} r^{2}-\frac{e^{2}}{4 \pi \varepsilon_{0} \varepsilon_{r} r} \\
\mathcal{H}_{m i x}(\overrightarrow{\mathbf{R}}, \overrightarrow{\mathbf{r}})= & \frac{e B}{M} \overrightarrow{\mathbf{r}} \times \overrightarrow{\mathbf{P}}_{c m} \\
& -2 \mu\left(\omega_{e}^{2}-\omega_{h}^{2}\right)\left(\overrightarrow{\mathbf{R}} \cdot \overrightarrow{\mathbf{r}}-\frac{\overrightarrow{\mathbf{R}}^{3} \cdot \overrightarrow{\mathbf{r}}}{R_{0}^{2}}\right) \\
& +\mu \omega_{r e l}^{2} \frac{\left[R^{2} r^{2}+2(\overrightarrow{\mathbf{R}} \cdot \overrightarrow{\mathbf{r}})^{2}\right]}{R_{0}^{2}} \\
& +2 \mu \frac{\left(m_{h}^{* 2} \omega_{e}^{2}-m_{e}^{* 2} \omega_{h}^{2}\right)}{M^{2}} \frac{\overrightarrow{\mathbf{R}} \cdot \overrightarrow{\mathbf{r}}}{R_{0}^{2}}
\end{aligned}
$$

where $\mu=\frac{m_{e}^{*} m_{h}^{*}}{M}$ is the electron-hole reduced mass and $M=m_{e}^{*}+m_{h}^{*}$ is the total mass. We have also introduced a center-of-mass frequency $\omega_{c m}=\sqrt{\frac{\left(m_{e}^{*} \omega_{e}^{2}+m_{h}^{*} \omega_{h}^{2}\right)}{M}}$ and a relative frequency $\omega_{\text {rel }}=\sqrt{\frac{m_{h}^{*} \omega_{e}^{2}+m_{e}^{*} \omega_{h}^{2}}{M}}$.

The main purpose in the change of variable above is to use the solutions of $H_{c m}$ and $H_{r e l}$ as a basis for solving the full Hamiltonian. Those solutions, i.e., labeled by $\psi_{\lambda}^{c m}(\overrightarrow{\mathbf{R}})$ and $\psi_{\lambda^{\prime}}^{r e l}(\overrightarrow{\mathbf{r}})$, can be solved by the series expansion method 26.27. Here, $\lambda=\left\{n_{c m}, l_{c m}\right\}$ and $\lambda^{\prime}=\left\{n_{r e l}, l_{\text {rel }}\right\}$ represent the quantum number pair of the radial quantum number $n$ and orbital angularmomentum quantum number $l$. Another advantage of the separation of center-of-mass and relative coordinates is that the negative Coulomb interaction $-\frac{e^{2}}{4 \pi \varepsilon_{0} \varepsilon_{r} r}$ appears in $H_{r e l}$ only, and the well-known poor-convergence of the parabolic basis is thus avoided when the characteristic scale of systems is beyond the effective Bohr 
radius 2328. We now search for the wave functions of the exciton in the form

$$
\Psi=\sum_{\lambda, \lambda^{\prime}} A_{\lambda, \lambda^{\prime}} \psi_{\lambda}^{c m}(\overrightarrow{\mathbf{R}}) \psi_{\lambda^{\prime}}^{r e l}(\overrightarrow{\mathbf{r}}) .
$$

Due to the cylindrical symmetry of the problem, the exciton wave functions can be labeled by the total orbital angular momentum $L=l_{c m}+l_{\text {rel }}$. To obtain the coefficients $A_{\lambda, \lambda^{\prime}}$, the total Hamiltonian is diagonalized in the space spanned by the product states $\psi_{\lambda}^{c m}(\overrightarrow{\mathbf{R}}) \psi_{\lambda^{\prime}}^{r e l}(\overrightarrow{\mathbf{r}})$. In the present calculations, we first solve the single particle problem of center-of-mass and relative Hamiltonians $H_{c m}$ and $H_{r e l}$, keep several hundreds of the single particle states, and then pick up the low-lying energy levels to construct several thousands of product states. Note that our numerical diagonalization scheme is very efficient and essentially exact in the sense that the accuracy can be improved as required by increasing the total number of selected product states.

Once the coefficients $A_{\lambda, \lambda^{\prime}}$ are obtained, one can calculate directly the measurable properties, such as the linear optical susceptibility of the nano-rings, whose imaginary part is related to the absorption intensity measured by optical emission experiments. In theory, the linear optical susceptibility is proportional to the dipole matrix elements between one electron-hole pair $j$ state and the vacuum state, which in turn is proportional to the oscillator strengths $F_{j}$. In the dipole approximation, it is given byes 30

$$
\begin{aligned}
F_{j} & =\left|\iint d \overrightarrow{\mathbf{R}} d \overrightarrow{\mathbf{r}} \Psi(\overrightarrow{\mathbf{R}}, \overrightarrow{\mathbf{r}}) \delta(\overrightarrow{\mathbf{r}})\right|^{2} \\
& =\left|\sum_{\lambda, \lambda^{\prime}} A_{\lambda, \lambda^{\prime}} \psi_{\lambda^{\prime}}^{r e l}(\mathbf{0}) \int d \overrightarrow{\mathbf{R}} \psi_{\lambda}^{c m}(\overrightarrow{\mathbf{R}})\right|^{2},
\end{aligned}
$$

where the factor $\psi_{\lambda^{\prime}}^{r e l}(\mathbf{0})$ and the integral over $\overrightarrow{\mathbf{R}}$ ensure that only the excitons with $L=0$ are created by absorbing photons. Therefore, the frequency dependence of the linear optical susceptibility $\chi(\omega)$ can be expressed as 28 B 30

$$
\chi(\omega) \propto \sum_{j} \frac{F_{j}}{\hbar \omega-E_{g}-E_{j}-i \Gamma}
$$

where $E_{g}$ and $E_{j}$ are the respective semiconducting band gap of InAs and energy levels of the exciton, and $\Gamma$ has been introduced as a phenomenological broadening parameter.

\section{NARROW WIDTH NANO-RINGS}

In order to explore the underlying physics of $\mathrm{ABE}$, we first consider a narrow-width nano-ring in which the analytical results are variable. We restrict ourselves with the condition $m_{e}^{*}=m_{h}^{*}=m^{*}$, but the general properties of nano-rings are not affected by this constraint. The technique employed in this section follows directly the previous works of Wendler, Fomin and Chaplik describing the rotating Wigner molecule behavior in quantum rings 14 16. 31 .

By introducing polar coordinates in the x-y plane $\overrightarrow{\mathbf{r}}_{i}=$ $\left(r_{i}, \varphi_{i}\right)$, the relative azimuthal coordinate $\varphi=\varphi_{e}-\varphi_{h}$, and the azimuthal coordinate $\Theta=\frac{\varphi_{e}+\varphi_{h}}{2}$ describing the motion of the electron-hole pair as a whole, the Hamiltonian (11) reads

$$
\begin{aligned}
\mathcal{H}= & \sum_{i=e, h}\left\{\left[-\frac{\hbar^{2}}{2 m^{*}}\left(\frac{\partial^{2}}{\partial r_{i}^{2}}+\frac{1}{r_{i}} \frac{\partial}{\partial r_{i}}\right)+U\left(r_{i}\right)\right]\right\} \\
& -\frac{\hbar^{2}}{2 m^{*}}\left\{\frac{1}{r_{e}^{2}}\left(\frac{\partial}{\partial \varphi}-i \frac{\Phi_{e}}{\Phi_{0}}\right)^{2}+\frac{1}{r_{h}^{2}}\left(\frac{\partial}{\partial \varphi}-i \frac{\Phi_{h}}{\Phi_{0}}\right)^{2}\right. \\
& \left.+\left(\frac{1}{r_{e}^{2}}+\frac{1}{r_{h}^{2}}\right) \frac{1}{4} \frac{\partial^{2}}{\partial \Theta^{2}}+\left(\frac{1}{r_{e}^{2}}-\frac{1}{r_{h}^{2}}\right) \frac{\partial}{\partial \varphi} \frac{\partial}{\partial \Theta}\right\} \\
& +V_{e-h}\left(\left|\overrightarrow{\mathbf{r}}_{e}-\overrightarrow{\mathbf{r}}_{h}\right|\right),
\end{aligned}
$$

where $\Phi_{e}=\pi B r_{e}^{2}$ and $\Phi_{h}=\pi B r_{h}^{2}$. For a narrow-width nano-ring, i.e., $W \ll R_{0}$, the radial motion is much faster than the azimuthal motions. Hence the radial motion is adiabatically decoupled from the azimuthal motions with the result

$$
\Psi\left(r_{1}, r_{2} ; \varphi, \Theta\right)=\sum_{n_{1}, n_{2}} \Xi_{n_{1}, n_{2}}\left(r_{1}, r_{2}\right) \psi_{n_{1}, n_{2}}(\varphi, \Theta)
$$

for the excitonic states. $\Xi_{n_{1}, n_{2}}\left(r_{1}, r_{2}\right)$ is a product of the corresponding single-particle wave functions $\chi_{n_{i}}\left(r_{i}\right)$ with eigenenergies $\varepsilon_{n_{i}}^{r a d}\left(n_{i}=0,1,2, \ldots\right)$, which describe the radial motion of electrons or holes with zero angular momentum. Because the single-particle wave functions are orthonormalized, the set $\left\{\Xi_{n_{1}, n_{2}}\left(r_{1}, r_{2}\right)\right\}$ forms a closure set of orthonormalized functions. The azimuthal wave function should satisfy the single-valuedness boundary conditions, i.e, $\psi_{n_{1}, n_{2}}\left(\varphi_{1}, \varphi_{2}\right)=\psi_{n_{1}, n_{2}}\left(\varphi_{1}+2 \pi, \varphi_{2}\right)=$ $\psi_{n_{1}, n_{2}}\left(\varphi_{1}, \varphi_{2}+2 \pi\right)=\psi_{n_{1}, n_{2}}\left(\varphi_{1}+2 \pi, \varphi_{2}+2 \pi\right)$, or in terms of the new variables $\varphi$ and $\Theta, \psi_{n_{1}, n_{2}}(\varphi, \Theta)=$ $\psi_{n_{1}, n_{2}}(\varphi+2 \pi, \Theta+\pi)=\psi_{n_{1}, n_{2}}(\varphi, \Theta+2 \pi)$.

As long as the above-stated criterion of the adiabatic approximation is satisfied, the excited states of radial motion lie high above the ground state. As a consequence we can restrict the consideration to the ground state of the radial motion because here only the lowest lying states are of interest. Thus we take only $n_{1}=n_{2}=0$ and substitute Eq. (7) into the Schrödinger equation $\mathcal{H} \Psi=E \Psi$ with the transformed Hamiltonian of Eq. (6), multiply both parts by $\Xi_{0,0}\left(r_{1}, r_{2}\right)$ and integrate over $r_{1}, r_{2}$. Hence the variables $\varphi$ and $\Theta$ become separated:

$$
\begin{aligned}
& \left\{-\frac{\hbar^{2}}{m^{*}}\left\langle\frac{1}{r_{1}^{2}}\right\rangle\left(\left(\frac{\partial}{\partial \varphi}-i \frac{\Phi}{\Phi_{0}}\right)^{2}+\frac{1}{4} \frac{\partial^{2}}{\partial \Theta^{2}}\right)\right. \\
& +\left\langle V_{e-h}\left(\sqrt{r_{1}^{2}+r_{2}^{2}-2 r_{1} r_{2} \cos \varphi}\right)\right\rangle \\
& \left.+2 \varepsilon_{0}^{r a d}-E\right\} \psi_{0,0}(\varphi, \Theta)=0,
\end{aligned}
$$


where $\Phi=\left\langle\Phi_{e}\right\rangle=\left\langle\Phi_{h}\right\rangle$ in the first order approximation, and \langle\rangle denotes the average with the radial wave functions

$$
\langle\ldots\rangle=\int d r_{1} r_{1} \int d r_{2} r_{2} \Xi_{0,0}^{*}\left(r_{1}, r_{2}\right) \ldots \Xi_{0,0}\left(r_{1}, r_{2}\right) .
$$

The magnetic flux in Eq. (8) can be removed by a gauge transformation with the price of introducing the twisted boundary conditions:

$$
\begin{aligned}
& \psi_{0,0}(\varphi, \Theta)=e^{i \frac{2 \pi \Phi}{\Phi_{0}}} \psi_{0,0}(\varphi+2 \pi, \Theta+\pi) \\
& \psi_{0,0}(\varphi, \Theta)=\psi_{0,0}(\varphi, \Theta+2 \pi)
\end{aligned}
$$

It is obvious from Eq. (8) that the relative azimuthal motion is decoupled from the azimuthal motion of the electron-hole pair as a whole. Therefore the azimuthal wave function can be represented in the form

$$
\psi_{0,0}(\varphi, \Theta)=\Phi_{j}^{p}(\varphi) Q_{\nu}(\Theta)
$$

where

$$
Q_{\nu}(\Theta)=\frac{1}{\sqrt{2 \pi}} e^{i \nu \Theta},
$$

and $\Phi_{j}^{p}(\varphi)$ is a solution of the equation

$$
\left\{-\frac{\hbar^{2}}{m^{*} R_{0}^{2}} \frac{\partial^{2}}{\partial \varphi^{2}}+V_{e-h}\left(2 R_{0}\left|\sin \frac{\varphi}{2}\right|\right)-\varepsilon_{j}^{r . a ., p}\right\} \Phi_{j}^{p}(\varphi)=0 .
$$

Here we have used

$$
\begin{aligned}
\left\langle\frac{1}{r_{1}^{2}}\right\rangle & =\frac{1}{R_{0}^{2}} \\
\left\langle V_{e-h}\left(\sqrt{r_{1}^{2}+r_{2}^{2}-2 r_{1} r_{2} \cos \varphi}\right)\right\rangle & =V_{e-h}\left(2 R_{0}\left|\sin \frac{\varphi}{2}\right|\right) .
\end{aligned}
$$

The index $p$ describes the possible symmetry types of $\Phi_{j}^{p}(\varphi)$ and can be specified below by the twisted boundary condition. In Fig. 2, we show three typical types of electron-hole interaction potentials $V_{e-h}\left(2 R_{0}\left|\sin \frac{\varphi}{2}\right|\right)$ with $2 \pi$ periodicity. In the view of the excitonic state, the potential should be strong enough to bind the electronhole pair, and then the relative azimuthal motion is strongly localized in each potential well. Thus the relative azimuthal wave function can be considered in the tight-binding-like form

$$
\Phi_{j}^{p}(\varphi)=\sum_{Q=-\infty}^{+\infty} \exp \left(i p \varphi_{Q}\right) \phi_{j}\left(\varphi-\varphi_{Q}\right),
$$

where $\varphi_{Q}=2 \pi Q ; Q=0, \pm 1, \pm 2, \ldots$. The wave function $\phi_{j}\left(\varphi-\varphi_{Q}\right)$ of a single well satisfies the equation

$$
\left\{-\frac{\hbar^{2}}{m^{*} R_{0}^{2}} \frac{\partial^{2}}{\partial \varphi^{2}}+V_{e-h}^{S W, Q}(\varphi)-\varepsilon_{j}^{r . a .}\right\} \phi_{j}\left(\varphi-\varphi_{Q}\right)=0,
$$

where $V_{e-h}^{S W, Q}(\varphi)$ is the potential of a given single well with minimum at $\varphi=\varphi_{Q}$ which coincides with the periodic potential $V_{e-h}\left(2 R_{0}\left|\sin \frac{\varphi}{2}\right|\right)$ in the region $\left|\varphi-\varphi_{Q}\right|<\pi$. Note that the tight-binding-like relative azimuthal wave function satisfies the Floquet-Bloch theorem, which is applicable to the periodic potential.

Combining Eqs. (12), (14) and (11) and substituting them into the twist boundary condition Eq. (10), we obtain

$$
\begin{aligned}
\nu & =\text { integer, } \\
p+\frac{\nu}{2}+\frac{\Phi}{\Phi_{0}} & =\text { integer } .
\end{aligned}
$$

It becomes obvious that $\nu$ describes the total angular momentum of excitons.

The tunneling through the potential from well to well leads to an explicit dependence of the energy levels $\varepsilon_{j}^{r . a ., p}$ on the symmetry parameter $p$ and thus results in energy bands as a function of the magnetic flux $\Phi$. In the tightbinding approximation, it is given by

$$
\varepsilon_{j}^{r . a ., p}=\varepsilon_{j}^{r . a .}-t_{0, j}-2 t_{1, j} \cos \left(2 \pi \frac{\Phi}{\Phi_{0}}+\pi \nu\right),
$$

where

$t_{0, j}=-\int d \varphi \phi_{j}\left(\varphi-\varphi_{0}\right)\left[V_{e-h}(\varphi)-V_{e-h}^{S W, 0}(\varphi)\right] \phi_{j}\left(\varphi-\varphi_{0}\right)$,
$t_{1, j}=-\int d \varphi \phi_{j}\left(\varphi-\varphi_{0}\right)\left[V_{e-h}(\varphi)-V_{e-h}^{S W, 0}(\varphi)\right] \phi_{j}\left(\varphi-\varphi_{1}\right)$.

It is obvious from Eq. (17) that the hopping integral $t_{1, j}$ determines the width of the energy band $\varepsilon_{j}^{r . a ., p}$ and causes the Aharonov-Bohm-like oscillation in energy levels. However, as follows from the detailed discussion of this effect below, $t_{1, j}$ is typically exponentially small compared with $\varepsilon_{j}^{r . a ., p}$. Only when $\varepsilon_{j}^{r . a}$. is very close to the maximum of interaction potential $V_{e-h}(\pi)$, does $t_{1, j}$ have a finite value. In other words, in order to access the measurable $A B$ effect, the bound electron and hole should have a possibility to tunnel in the opposite directions and meet each other "on the opposite side of the nano-ring" $(\varphi=\pi)$.

Using the wave function (11) of a state with a fixed angular momentum $\nu$ in the Schrödinger equation (8), we finally obtain the eigenenergies of the full Hamiltonian,

$$
E_{0,0, p, j, \nu}=2 \varepsilon_{0}^{r a d}+\varepsilon_{j}^{r . a ., p}+\frac{\hbar^{2} \nu^{2}}{4 m^{*} R_{0}^{2}},
$$

and the expression for the oscillation strengths

$$
F_{j}=\frac{\left|\Phi_{j}^{p}(0)\right|^{2}}{\int_{-\pi}^{+\pi} d \varphi\left|\Phi_{j}^{p}(\varphi)\right|^{2}} \delta_{\nu, 0}
$$

In the following we consider the detailed examples for three typical electron-hole interaction potentials, restricting ourselves with $\nu=0$. 


\section{A. short ranged potential}

We use the $\delta$ potential to simulate the short ranged potential, i.e. $V_{e-h}\left(2 R_{0}\left|\sin \frac{\varphi}{2}\right|\right)=-2 \pi V_{0} \delta\left(2\left|\sin \frac{\varphi}{2}\right|\right)$, and choose $V_{e-h}^{S W, Q}(\varphi)=-2 \pi V_{0} \delta\left(\varphi-\varphi_{Q}\right)$ (see Fig. (2a)). By solving the one-dimensional Schrödinger equation

$$
\left\{-\frac{\hbar^{2}}{m^{*} R_{0}^{2}} \frac{\partial^{2}}{\partial \varphi^{2}}-2 \pi V_{0} \delta\left(\varphi-\varphi_{Q}\right)-\varepsilon_{j}^{r . a}\right\} \phi_{j}\left(\varphi-\varphi_{Q}\right)=0,
$$

we obtain one bound eigenstate with the eigenfunction and eigenenergy

$$
\begin{aligned}
\phi_{0}\left(\varphi-\varphi_{Q}\right) & =\sqrt{k} \exp \left(-k\left|\varphi-\varphi_{Q}\right|\right), \\
\varepsilon_{0}^{r . a} & =-\frac{\hbar^{2} k^{2}}{m^{*} R_{0}^{2}}=-\pi V_{0} k
\end{aligned}
$$

respectively. Here $k \equiv \frac{\pi V_{0}}{\left(\frac{\hbar^{2}}{m^{*} R_{0}^{2}}\right)} \gg 1$. Substituting the single well wave function (21) in Eq. (18), in the limit of large $k$, we get the result

$$
\begin{aligned}
& t_{0, j} \approx 4 \pi V_{0} k \exp (-4 \pi k), \\
& t_{1, j} \approx 2 \pi V_{0} k \exp (-2 \pi k),
\end{aligned}
$$

and

$$
\varepsilon_{0}^{r . a ., p} \approx-\pi V_{0} k\left[1+4 \cos \left(\frac{2 \pi \Phi}{\Phi_{0}}\right) \exp (-2 \pi k)\right] .
$$

Note that the expression for $\varepsilon_{0}^{r . a ., p}$ agrees exactly with that obtained by Römer and Raikh (Eq. (19) in Ref. (22)).

\section{B. harmonic potential}

In this case the electron-hole interaction potential and single well potential are given by

$$
\begin{aligned}
V_{e-h}\left(2 R_{0}\left|\sin \frac{\varphi}{2}\right|\right) & =\frac{m^{*} R_{0}^{2}}{4} \omega^{2}\left(2 \sin \frac{\varphi}{2}\right)^{2}, \\
V_{e-h}^{S W, Q}(\varphi) & =\frac{m^{*} R_{0}^{2}}{4} \omega^{2}\left(\varphi-\varphi_{Q}\right)^{2},
\end{aligned}
$$

respectively. A Schematic plot of these two potentials is shown in Fig. (2b). Once again we should solve the one-dimensional Schrödinger equation

$$
\left\{-\frac{\hbar^{2}}{2 I} \frac{\partial^{2}}{\partial \varphi^{2}}+\frac{I \omega^{2}}{2}\left(\varphi-\varphi_{Q}\right)^{2}-\varepsilon_{j}^{r . a .}\right\} \phi_{j}\left(\varphi-\varphi_{Q}\right)=0
$$

where $I=\frac{m^{*} R_{0}^{2}}{2}$. It is nothing but an equation describing a shifted harmonic oscillator. The eigenfunctions and eigenenergies are thus given by

$$
\begin{aligned}
\phi_{j}\left(\varphi-\varphi_{Q}\right)= & \frac{1}{\sqrt{2^{j} j ! \pi^{1 / 2} \xi}} \exp \left[-\frac{1}{2}\left(\frac{\varphi-\varphi_{Q}}{\xi}\right)^{2}\right] \\
& \times H_{j}\left(\frac{\varphi-\varphi_{Q}}{\xi}\right), \\
\varepsilon_{j}^{r . a .}= & \hbar \omega\left(j+\frac{1}{2}\right), \quad j=0,1,2, \ldots
\end{aligned}
$$

In Eq. (24), $\xi \equiv\left(\hbar \omega / 2\left(\frac{\hbar^{2}}{m^{*} R_{0}^{2}}\right)\right)^{-1 / 2} \ll 1$ is the typical width of the single well wave function and $H_{j}(x)$ is Hermite polynomial 32 . By substituting Eq. (21) in Eqs. (18) and (17), in the limit of small $\xi$, the final result is

$$
\begin{aligned}
& t_{0, j} \approx \alpha_{j} \hbar \omega \\
& t_{1, j} \approx(-)^{j} \beta_{j} \exp \left(-\frac{\pi^{2}}{\xi^{2}}\right) \hbar \omega,
\end{aligned}
$$

and

$$
\begin{aligned}
\varepsilon_{j}^{r . a ., p} \approx & \hbar \omega\left[\left(j+\frac{1}{2}\right)\right. \\
& \left.-\alpha_{j}+(-)^{j+1} 2 \beta_{j} \exp \left(-\frac{\pi^{2}}{\xi^{2}}\right) \cos \left(\frac{2 \pi \Phi}{\Phi_{0}}\right)\right],
\end{aligned}
$$

where

$$
\begin{aligned}
& \alpha_{j}=\frac{\xi^{2}}{24} \frac{1}{2^{j} j ! \pi^{1 / 2}} \int_{-\infty}^{+\infty} d x x^{4} H_{j}^{2}(x) \exp \left(-x^{2}\right), \\
& \beta_{j}=\frac{\left(\pi^{2}-4\right)}{2^{j+1} j ! \xi^{2}} H_{j}^{2}\left(\frac{\pi}{\xi}\right) .
\end{aligned}
$$

\section{Coulomb interaction potential}

Let us consider the more realistic Coulomb interaction potential which has the form (see also Fig. (2c))

$$
\begin{aligned}
V_{e-h}\left(2 R_{0}\left|\sin \frac{\varphi}{2}\right|\right) & =-\frac{e^{2}}{4 \pi \varepsilon_{0} \varepsilon_{r} R_{0} 2\left|\sin \frac{\varphi}{2}\right|}, \\
V_{e-h}^{S W, Q}(\varphi) & =-\frac{e^{2}}{4 \pi \varepsilon_{0} \varepsilon_{r} R_{0}\left|\varphi-\varphi_{Q}\right|}
\end{aligned}
$$

We now face to solve the Schrödinger equation for an one-dimensional exciton,

$\left\{-\frac{\hbar^{2}}{m^{*} R_{0}^{2}} \frac{\partial^{2}}{\partial \varphi^{2}}-\frac{e^{2}}{4 \pi \varepsilon_{0} \varepsilon_{r} R_{0}\left|\varphi-\varphi_{Q}\right|}-\varepsilon_{j}^{r . a .}\right\} \phi_{j}\left(\varphi-\varphi_{Q}\right)=0$.

The ground state solution gives a logarithmically divergent eigenenergy $\left(\varepsilon_{j}^{r . a .} \rightarrow-\infty\right)$ and the normalized eigenfunction behaves like a $\delta$ function 33 . In this sense, the electron-hole pair tends to bind extremely tightly, and is prevented to tunnel through the mean Coulomb potential barrier to induce any measurable AB effect. This 
situation is also expected if one introduces a finite but small ring width $W$. On the other hand, each excited state of one-dimensional exciton is two-fold degenerate and classified further by the parity symmetry parameter $\theta(\theta=0,1$ corresponds to even and odd parity symmetry, respectively.). The detailed eigenfunctions can be constructed from the radial wave function of a hydrogen atom with zero angular momentum, and are given by

$$
\begin{aligned}
\phi_{j \theta}\left(\varphi-\varphi_{Q}\right)= & \left\{\begin{array}{ll}
\psi_{j}\left(\varphi-\varphi_{Q}\right) & \varphi>\varphi_{Q} \\
(-)^{\theta} \psi_{j}\left(\varphi-\varphi_{Q}\right) & \varphi<\varphi_{Q}
\end{array}, \quad j=1,2, \ldots\right. \\
\psi_{j}\left(\varphi-\varphi_{Q}\right)= & \left(\frac{2}{j^{3} a^{3}}\right)^{1 / 2}\left|\varphi-\varphi_{Q}\right| \exp \left(-\frac{\left|\varphi-\varphi_{Q}\right|}{j a}\right) \\
& \times F\left(-j+1,2, \frac{2\left|\varphi-\varphi_{Q}\right|}{j a}\right),
\end{aligned}
$$

where $a \equiv 2\left(\frac{\hbar^{2}}{m^{*} R_{0}^{2}}\right) /\left(\frac{e^{2}}{4 \pi \varepsilon_{0} \varepsilon_{r} R_{0}}\right) \ll 1$ and $F$ is a hypergeometric function 32 . The corresponding eigenenergies have the form

$$
\varepsilon_{j \theta}^{r . a .}=-\frac{1}{a j^{2}} \frac{e^{2}}{8 \pi \varepsilon_{0} \varepsilon_{r} R_{0}},
$$

similar to the energy spectra of a hydrogen atom. It should be pointed out that the eigenfunctions vanish at $\varphi=\varphi_{Q}$ since the singular and non-integrable Coulomb potential acts_as an impenetrable barrier for both electron and hole 3 . This effect gives an exponentially small oscillator strength for the excited states (see Eq. (20)) and leads to the rapid damping of the low-lying excitonic transitions in the linear optical susceptibility for a finite width nano-ring as shown in the next section. The hopping integrals in Eq. (18) are tedious to integrate, and in the first order of small- $a$ limit the final answer is

$$
\begin{aligned}
& t_{0, j} \approx \alpha_{j} \frac{e^{2}}{8 \pi \varepsilon_{0} \varepsilon_{r} R_{0}}, \\
& t_{1, j} \approx(-)^{\theta} \beta_{j} \exp \left(-\frac{2 \pi}{j a}\right) \frac{e^{2}}{8 \pi \varepsilon_{0} \varepsilon_{r} R_{0}},
\end{aligned}
$$

and

$$
\begin{aligned}
\varepsilon_{j \theta}^{r . a ., p} \approx & -\frac{e^{2}}{8 \pi \varepsilon_{0} \varepsilon_{r} R_{0}}\left[\frac{1}{a j^{2}}\right. \\
& \left.+\alpha_{j}+(-)^{\theta} 2 \beta_{j} \exp \left(-\frac{2 \pi}{j a}\right) \cos \left(\frac{2 \pi \Phi}{\Phi_{0}}\right)\right]
\end{aligned}
$$

where

$$
\begin{aligned}
& \alpha_{j}=\frac{j a}{48} \int_{0}^{+\infty} d x x^{3}[F(-j+1,2, x)]^{2} \exp (-x), \\
& \beta_{j}=\frac{2 \pi(\pi-2)}{j^{3} a^{3}}(2 \pi+j a)[F(-j+1,2,2 \pi / j a)]^{2} .
\end{aligned}
$$

At the end of this section, we would like to emphasize the following features: (i) For all the cases we have considered, both for ground state and excited states the hopping integral $t_{1, j}$ is typically exponentially small compared with $\varepsilon_{j}^{r . a}$, and the exponent factor is determined by the ratio of electronhole interaction potential and the characteristic energy of rotation mode. This prediction is in apparent contradiction to the recent claim of Römer and Raikh22, which predicted that the ABE oscillations are found to be much more easily in the case of excited states, and are not exponentially small. This discrepancy might arise from the definition of "excited states" of excitons. Recall that there is only one bound state for electron-hole pair with short-ranged interaction potential (at least with $\delta$ potential). Therefore, the "excited states" said by Römer and Raikh is more appropriate to be regarded as "excited states" of free electrons or holes instead of excitons.

(ii) In the case of the Coulomb interaction potential, there is no $\mathrm{ABE}$ for the ground excitonic state due to the intrinsic divergence of the ground state energy of onedimensional excitons 33 .

(iii) On the other hand, in order to observe the AB effect in excited states, the single well eigenenergies $\varepsilon_{j}^{r . a .}$ should be tuned closely to the potential maximum $V_{e-h}(\pi)$. Because of the particular properties of the energy spectra in the presence of Coulomb interaction, i.e., $\varepsilon_{j \theta}^{r . a .} \propto-1 / j^{2}$, the Coulomb interaction potential is appropriate to observe the $\mathrm{AB}$ effect in excited states compared with other potentials. For example, for a narrow-width nano-ring with $R_{0}=20 \mathrm{~nm}$, by setting $\varepsilon_{j \theta}^{r . a .} \sim-\frac{e^{2}}{8 \pi \varepsilon_{0} \varepsilon_{r} R_{0}}$, a rough estimation gives $j \sim 1$, which agrees well with the numerical results for lighthole-excitons in InAs nano-rings as shown below.

\section{NUMERICAL RESULTS AND DISCUSSIONS}

In order to understand the influence of a finite ring width on the $\mathrm{AB}$ effect, let us now discuss the numerical results for a realistic self-assembled semiconducting InAs nano-ring. In the following we restrict ourselves in the subspace $L=0$. We have taken the parameters $m_{e}^{*}=0.067 m_{e}$, the effective mass of the light hole $m_{l h}^{*}=0.099 m_{e}$, the effective mass of the heavy hole $m_{h h}^{*}=0.335 m_{e}\left(m_{e}\right.$ is the bare mass of a single electron) and $\varepsilon_{0}=12.4$, which are appropriate to InAs material 35, 10. The electron and hole are considered to be confined in a confinement potential with the same strength, i.e., $m_{e}^{*} \omega_{e}^{2}=m_{h}^{*} \omega_{h}^{2}$. The ring radius $R_{0}$ and characteristic frequency of the radial confinement $\hbar \omega_{e}$ are chosen to be $20 \mathrm{~nm}$ and $14 \mathrm{meV}\left(\hbar \omega_{e}=14 \mathrm{meV}\right.$ corresponds to $W=10 \mathrm{~nm}$ ), respectively, simulating a recent experimental results $\Delta E_{m} \sim 5 \mathrm{meV}$ and $\Delta E_{n} \sim 20-25$ $\mathrm{meV}$ in InAs nano-rings $24, \Delta E_{m}$ is the energy level spacing between the single electron states with different orbital angular momentum $m$ and the same radial quantum number $n$, while $\Delta E_{n}$ corresponds to the energy spacing with different radial quantum number $n$ and the same 
angular momentum $m$. In the calculations, we use effective atomic units in which the length unit $a_{B}^{*}$ is a factor $\frac{\varepsilon_{r}}{\mu}$ times the Bohr radius $a_{B}$, and the energy is given in effective hartrees, $H_{a}^{*}=\left(\mu / \varepsilon_{r}^{2}\right) \times 1$ hartree (For the heavy-hole-exciton, for example, the length and energy units then scale to $a_{B}^{*}=11.8 \mathrm{~nm}$ and $H_{a}^{*}=10.0 \mathrm{meV}$ ). For $R_{0}=20 \mathrm{~nm}$, the universal flux quantum $\Phi_{0}$ corresponds to the magnetic field $B \approx 4.1 \mathrm{~T}$.

By probing of the structure of the numerical excitonic wave functions with the use of the conditional probability distribution (CPD) and pair-correlation function (PCF) 35. 36 , we first study the hopping integral $t_{1, j}$ (or the tunneling possibility) which plays the most essential role in the $\mathrm{AB}$ effect. Denoting the numerical wave function of excitons by $\Psi\left(\mathbf{r}_{e}, \mathbf{r}_{h}\right)$ (see Eq. (3), which is the summation of the product of the centre-of-mass and relative wave functions), we define the usual PCF as

$$
G(v)=2 \pi \iint \delta\left(\mathbf{r}_{e}-\mathbf{r}_{h}-\mathbf{v}\right)\left|\Psi\left(\mathbf{r}_{e}, \mathbf{r}_{h}\right)\right|^{2} d \mathbf{r}_{e} d \mathbf{r}_{h},
$$

and the CPD for finding the electron at $\mathbf{v}$ given that the hole is at $\mathbf{r}_{h}=\mathbf{v}_{0}$ as

$$
\mathcal{P}\left(\mathbf{v} \mid \mathbf{r}_{h}=\mathbf{v}_{0}\right)=\frac{\left|\Psi\left(\mathbf{v}, \mathbf{r}_{h}=\mathbf{v}_{0}\right)\right|^{2}}{\int d \mathbf{r}_{e}\left|\Psi\left(\mathbf{r}_{e}, \mathbf{r}_{h}=\mathbf{v}_{0}\right)\right|^{2}} .
$$

Note that for the exact $\Psi$ in the case of a circularly symmetric confinement, the PCF turn out to be circularly symmetric.

With the above, we solved for heavy-hole-exciton energy spectra and wave functions for $W=10 \mathrm{~nm}$. The selected PCF's and CPD's are displayed in Fig. 3. For the ground state in Fig. (3a), the CPD exhibits a highly localized electron density around the hole, namely, the electron and hole tends to bind tightly with each other. The above picture is also reflected in the PCF, which shows a rapid delay as the distance between electron and hole increases. This behaviour agrees well with the rigorous analysis present earlier in Sec IIIC. In contrast to the ground state, in Figs. (3b) and (3c) the CPD's for the fifth and sixth excited states exhibit a well-developed local maximum probability for finding the electron around the diametrically opposite point $(-8,0)$, suggesting that the electron and hole have a possibility to tunnel through the Coulomb potential barrier in the opposite directions. Observe also that the PCF's in Figs. (3b) and (3c) reveal a clear local maximum at $v \sim 35 \mathrm{~nm}$. Moreover, in the case of the eighth and ninth excited states (see Fig. 4 ), one can even identify a larger hopping integral. The remarkable emergence of the hopping integral for lowlying excited excitonic states provides us a possibility for observing a non-vanishing $\mathrm{AB}$ effect.

Figs. (5a) and (5b) display the low-lying energy levels as a function of the magnetic field for heavy-hole-exciton and light-hole-exciton. As can be seen immediately, the most obvious feature is the $\mathrm{AB}$ oscillations of some lowlying energy levels (indicated by an arrow) with a period $B \approx 4.1 \mathrm{~T}$ or a flux quantum $\Phi_{0}$, as expected. However, the $\mathrm{AB}$ oscillations are not very close to sinusoidal as illustrated by Eq. (17), due to the finite width of nano-rings. The typical amplitude of those oscillations is about $3 \mathrm{meV}$, which is much larger than the extremely narrow luminescence line-width in a single InAs nanoring 37 , and in view of this it might be sensitive to be detected by present optical emission techniques. Comparing Figs. (5a) and (5b), it is seen that the energy levels of light-hole-exciton show a relatively pronounced $\mathrm{AB}$ effect. Even the energy level of the first excite state of lighthole-exciton tends to oscillate with magnetic field. This is due the comparable effective mass between electron and light-hole, which suppresses the electron-hole binding (i.e., the much higher ground state energy of lighthole-exciton compared with that of heavy-hole-exciton) and thus enhances the $\mathrm{AB}$ effect.

Another noticeable features shown in Figs. (5a) and (5b) include: (i) There are many anticrossings in the energy levels which is caused by level repulsions. (ii) Contrary to conventional quantum dots, when the magnetic field is increased it seems unlikely to form any Landau levels. (iii) In the high magnetic field, the energy level of the ground state and first excited state shows a slight blue shift, due to the diamagnetic effect for each charge carrier which pushes all the relative energies upwards. This behavior is qualitatively similar to the excitons in quantum dots. Note that those blue shifts are observed in a recent experiment 38 .

The behavior of the oscillator strengths for heavy-holeexciton and light-hole-exciton as a function of the magnetic field is present in Figs. (6a)-(6d). It is readily seen that periodic oscillations take place in the oscillator strengths for the selected low-lying excited states. Though those oscillations are not regular in shape and not close to the expected sinusoidal, they are still supposed to be caused by $\mathrm{AB}$ phenomena. In contrast, for the ground state, the oscillator strength increase monotonously with the magnetic field. The result is in line with the blue shifts of the ground-state energies, for example, the increasing magnetic field increases the confinement, decreases the separation between electron and hole and in turn yields the enhancement of oscillator strengths. Furthermore, the overall magnitude of the oscillator strength of the ground state is approximately one order larger that of low-lying excited states, in good agreement with the analysis present earlier that the oscillator strengths for excited states are exponentially small for ideal one-dimensional nano-rings.

To support the experimental relevance of our results and better understand the periodic, Aharonov-Bohmtype oscillation in energy spectra, the imaginary part of linear optical susceptibility is plotted as a function of frequency $\omega$ for different magnetic fields in Figs. (7a)(7d), where a broadening parameter $\Gamma=0.5 \mathrm{meV}$ is used. Those curves represent all the possible transitions of ex- 
citonic states which would be measurable via photoluminescence excitation measurements (PLE). As expected, the periodic oscillations at some low-lying energy levels $(\omega \sim 30 \mathrm{meV})$ are well-reflected. However, the amplitude of those oscillations is very small compared with that of the fundamental transition because of the weak oscillator strengths of low-lying excited states. In fact, it is an indication of the delicate nature of the $\mathrm{AB}$ effect, which suggests that a highly sensitive technique is needed in experiment to access these coherent $\mathrm{AB}$ oscillations.

It is important to point out that our results mentioned above are in apparent contradiction to the prediction given by Song and Ulloa in their recent works, in which they claimed that the excitons in nano-rings behave to a great extent as those in quantum dots of similar dimensions and the $\mathrm{AB}$ oscillation of exciton characteristics predicted for one-dimensional rings are found to not be present in finite-width systems 23 . Here we indeed observe the ABE of some of low-lying exciton states, which is suggested by the CPD's and PCF's, illustrated by the energy spectra and oscillator strengths and finally confirmed by the imaginary part of linear optical susceptibility. Therefore, we believe that the finite but small width of the nano-rings will not suppress the ABE so greatly. Since the parameters used by Song and Ulloa are quite similar to ours, we suggest that the discrepancy might originate from the following reasons:

(i) In their paper, the negative Coulomb interaction term has been simply treated as a perturbation within a parabolic basis. However, just as the Authors mentioned, when the characteristic scale of systems is beyond the effective Bohr radius, the known poor-convergence of the parabolic basis might lead to unreliable results, especially for the excited states.

(ii) For the radius of ring in the Fig. 4 in Ref. (23), $R_{0}=24 \mathrm{~nm}$, the periodicity of $\mathrm{AB}$ oscillation is expected to be given by a period $\Delta B \approx 2.9 \mathrm{~T}$. However, the $\mathrm{Au}-$ thors only show the results for different magnetic fields increased on steps of $5.0 \mathrm{~T}$. With so large interval, one might miss the AB oscillations completely..

We next turn out to investigate the size effect by tunning the ring width. As an illustration, the low-lying energy levels of the heavy-hole exciton for a narrow ring width $W=8 \mathrm{~nm}$, shown as curve (a) in Fig. 8, is compared to the wide ring width case with $W=14,20$ and $30 \mathrm{~nm}$ (shown in Fig. 8 as curves (b), (c) and (d), respectively). It is readily seen that as the ring width increases, the AB oscillation pattern is gradually destroyed. The disappearance of $\mathrm{AB}$ effect arises from the destruction of the non-simply-connected geometry of nano-rings, since the increasing width lowers the soft confinement potential barrier at the ring center and in turn yields a high possibility for carriers to reside. For $W=30 \mathrm{~nm}$, which is comparable to the ring diameter $d=2 R_{0}=40 \mathrm{~nm}$, the main characteristic of the energy spectra resembles that of quantum dots 3941 . In Fig. 9, the corresponding imaginary part of linear optical susceptibility is plotted as a function of frequency. Comparison of the case of $W=8$ nm in Fig. (9a) with the wide width limit (Fig. (9d)) is instructive, since it exhibits a distinctive difference of the excitonic optical properties between nano-rings and quantum dots. Unlike the conventional quantum dots, in which the low-lying exciton state transitions have the same amplitudes and are nearly equally distributed (a reflection of excitations involving the exciton ground state and various center-of-mass replicas without altering the ground state of the relative coordinate), the low-lying transitions of nano-rings show a rapid dampen with frequency and theirs positions are not periodic. This difference is a refection of the anisotropic confinement of nano-rings 23: Since the exciton is confined in a quasi-onedimensional system, its center-of-mass degree of freedom is greatly suppressed and its relative motion becomes dominant (i.e. the general properties of one-dimensional exciton is expected), thus resulting in the destruction of the regular patterns observed in quantum dots. It is interesting to note that this distinctive difference is indeed observed by a recent experiment given by Pettersson et al.24. 42.43 .

\section{CONCLUSION}

In conclusion, we have studied the magnetic field effect on excitons in an InAs nano-ring based on a simple model Hamiltonian. By numerical diagonalization, we calculate the low-lying energy levels, oscillator strengths, and the corresponding linear optical susceptibility. A periodic, Aharonov-Bohm-type oscillation is clearly revealed in some low-lying energy levels and linear optical susceptibility curves for a realistic self-assembled semiconducting InAs nano-ring. This AB effect is well interpreted with the rigorous analysis for narrow-width nano-rings.

In a recent work, Warburton et al. presented a beautiful experiment of optical emission in a single chargetunable nano-ring 37 . They studied the role of multiplycharged exciton complexes with no applied magnetic field and found a shellstructure in energy similar to that of quantum dots 445 . Therefore, encouraged by the rapid developed nano-techniques for detection, i.e., the achievement of extremely narrow and temperature insensitive luminescence lines from a single InAs nano-ring in GaAs, we hope that our predictions of the ABE effects can be confronted in experiments in the future.

\section{ACKNOWLEDGMENTS}

We would like to thank Dr. H. Pettersson for helpful discussion and Dr. A. V. Chaplik for sending a copy of the paper (Ref.21). This research is financially supported by the NSF-China (Grant No.19974019) and China's "973" program. 
${ }^{1}$ A. Lorke and R. J. Luyken, Physica B 256, 424 (1998).

${ }^{2}$ A. Lorke, R. J. Luyken, M. Fricke, J. P. Kotthaus, G. Medeiros-Ribeiro, J. M. Garcia, and P. M. Petroff, Microelectronic Engineering 47, 95 (1999).

${ }^{3}$ A. Lorke, R. J. Luyken, A. O. Govorov, J. P. Kotthaus, J. M. Garcia, and P. M. Petroff, Phys. Rev. Lett. 84, 2223 (2000).

${ }^{4}$ D. Mailly, C. Chapelier, and A. Benoit, Phys. Rev. Lett. 70, 2020 (1993).

${ }^{5}$ A. Emperador, M. Pi, M. Barranco, and A. Lorke, Phys. Rev. B 62, 4573 (2000).

${ }^{6}$ P. Borrmann and J. Harting, eprint cond-mat/0008464 (2000).

${ }^{7}$ M. Koskinen, M. Manninen, B. Mottelson and S. M. Reimann, eprint cond-mat/0004095 (2000).

${ }^{8}$ A. Bruno-Alfonso and A. Latgé, Phys. Rev. B 61, 15887 (2000).

${ }^{9}$ X. S. Chen, Z.-Y. Deng, W. Lu, and S. C. Shen, Phys. Rev. B 61, 2008 (2000).

${ }^{10} \mathrm{H}$. Hu, J. -L. Zhu, and J. J. Xiong, eprint condmat/0005520, to be published in Phys. Rev. B (2000).

${ }^{11}$ J. Yi, H. Doh, and S.-I. Lee, Phys. Rev. B 59, 12192 (1999).

${ }^{12}$ E. Ben-Jacob, F. Guinea, Z. Hermon, and A. Shnirman, Phys. Rev. B 57, 6612 (1998).

${ }^{13}$ C. A. Stafford and D. F. Wang, Phys. Rev. B 56, R4383 (1997).

${ }^{14}$ L. Wendler and V. M. Fomin, Phys. Rev. B 51, 17814 (1995).

${ }^{15}$ L. Wendler, V. M. Fomin, A. V. Chaplik, and A. O. Govorov, Phys. Rev. B 54, 4794 (1996).

${ }^{16}$ L. Wendler, V. M. Fomin, A. V. Chaplik, and A. O. Govorov, Z. Phys. B 102, 211(1996).

17 T. Chakraborty and P. Pietiläinen, Phys. Rev. B 50, 8460 (1994).

${ }^{18}$ V. Gudmundsson and Á. Loftsdóttir, Phys. Rev. B 50, 17433 (1994)

${ }^{19}$ V. Halonen, P. Pietiläinen, and T. Chakraborty, Europhys. Lett. 33, 377 (1996).

${ }^{20}$ K. Niemelä, P. Pietiläinen, P. Hyvönen and T. Chakraborty, Europhys. Lett. 36, 533 (1996).

${ }^{21}$ A. Chaplik, JETP Lett. 62, 900 (1995).

${ }^{22}$ R. A. Römer and M. E. Raikh, Phys. Rev. B 62, 7045 (2000).

23 J. Song and S. E. Ulloa, eprint cond-mat/0008407 (2000).

${ }^{24}$ H. Pettersson, R. J. Warburton, A. Lorke, K. Karrai, J. P. Kotthaus, J. M. Garcia, and P. M. Petroff, Physica E 6, $510(2000)$.

${ }^{25} \mathrm{H}$. Hu et al., unpublished.

${ }^{26}$ J. -L. Zhu, J. J. Xiong, and B. -L. Gu, Phys. Rev. B 41, $6001(1990)$

27 J. -L. Zhu, Z. Q. Li, J. Z. Yu, K. Ohno, and Y. Kawazoe, Phys. Rev. B 55, 1 (1997).

${ }^{28}$ J. Song and S. E. Ulloa, Phys. Rev. B 52, 9015 (1995).

${ }^{29}$ G. W. Bryant, Phys. Rev. B 37, 8763 (1988).

${ }^{30}$ W. Que, Phys. Rev. B 45, 11036 (1992).

${ }^{31}$ Ifon completion of this work, we finally obtained the Ref. (21), in which the main result is similar to the one in Sec. IIIC.

${ }^{32}$ Handbook of Mathematical Functions, M. Abramowitz and I. A. Stegun (Dover, New York, 1972).
${ }^{33}$ R. Loudon, Am. J. Phys. 27, 649 (1959).

${ }^{34}$ M. Andrews, Am. J. Phys. 44, 1064 (1976).

${ }^{35}$ C. Yannouleas and U. Landman, Phys. Rev. B 61, 15895 (2000).

${ }^{36}$ C. Yannouleas and U. Landman, Phys. Rev. Lett. 85, 1726 (2000).

${ }^{37}$ R. J. Warburton, C. Schäflein, D. Haft, F. Bickel, A. Lorke, K. Karrai, J. M. Garcia, W. Schoenfeld, and P. M. Petroff, Nature 405, 926 (2000).

${ }^{38} \mathrm{H}$. Pettersson, private communication.

${ }^{39}$ V. Halonen, T. Chakraborty and P. Pietiläinen, Phys. Rev. B 45, 5980 (1992).

${ }^{40}$ A. Wojs and P. Hawrylak, Phys. Rev. B 51,10880 (1995).

${ }^{41}$ P. Hawrylak, Phys. Rev. B 60, 5597 (1999).

${ }^{42}$ H. Hu, G. -M Zhang, J. -L. Zhu, and J. J. Xiong, eprint cond-mat/0010186, to be published in Phys. Rev. B (2000).

${ }^{43}$ H. Hu, D. J. Li, J. -L. Zhu, and J. J. Xiong, eprint condmat/0009044, to be published in J. Phys: Condens. Matter (2000).

${ }^{44}$ M. Bayer, O. Stern, P. Hawrylak, S. Fafard, and A. Forchel, Nature 405, 923 (2000).

${ }^{45}$ P. Hawrylak, G. A. Narvaez, M. Bayer, and A. Forchel, Phys. Rev. Lett. 85, 389 (2000).

\section{Figures Captions}

Fig.1. Schematic geometry of InAs nano-rings with radius $R_{0}=20 \mathrm{~nm}$ and width $W=10 \mathrm{~nm}$ (a) or $7 \mathrm{~nm}$ (b) for electrons. $m_{e}^{*}=0.067 m_{e}$.

Fig.2. Scheme of different interaction potentials $V_{e-h}(\varphi)$ as a function of the relative azimuthal coordinate $\varphi=$ $\varphi_{e}-\varphi_{h}$. (a) Short-ranged form $-2 \pi V_{0} \delta\left(2 \sin \frac{\varphi}{2}\right)$, (b) parabolic form $\frac{\mu \omega^{2} \rho^{2}}{4}\left(2 \sin \frac{\varphi}{2}\right)^{2}$ and (c) Coulomb form $-\frac{e^{2}}{4 \pi \varepsilon_{0} \varepsilon_{r} \rho \mid 2 \sin \frac{\varphi}{2}}$. The potential of a given single well with minimum at $\varphi=0, V_{e-h}^{S W, 0}(\varphi)$ is delineated by the thick solid line. Some low-lying energy levels for each well are also indicated.

Fig.3. The conditional probability distributions (CPD's) and pair-correlation functions (PCF's) shown, respectively, on the left and right of each of the subplots [labeled by (a)-(c)], for heavy-hole exciton in an InAs nano-ring with radius $R_{0}=20 \mathrm{~nm}$ and width $W=10 \mathrm{~nm}$. Each CPD is expressed in a logarithmic intensity scale with the range from 0.01 (black) to 1.0 (white). The heavyhole is fixed at $\mathbf{v}_{0}=(20,0)$. (a) The ground state, (b) the fifth and (c) sixth excited states.

Fig.4. The conditional probability distributions (CPD's) for the eighth (upper panel) and ninth (lower panel) excited states. Other parameters are the same as in Fig. 3.

Fig.5. The low-lying energy levels of InAs nano-rings with radius $R_{0}=20 \mathrm{~nm}$ and width $W=10 \mathrm{~nm}$ as a function of the magnetic field for heavy-hole (a) and lighthole (b) excitons. As indicated by the arrow, a periodic, 
Aharonov-Bohm-type oscillation in some low-lying energy levels takes place.

Fig.6. The oscillator strengths of InAs nano-rings as a function of the magnetic field for heavy-hole (upper panel, (a) and (b)) and light-hole (lower panel, (c) and (d)) excitons. (a) (c) for ground state and (b) (d) for excited states (the solid, dashed and dotted lines correspond to the fourth, fifth and sixth excited states).

Fig.7. Imaginary part of linear optical susceptibility as a function of frequency for different magnetic fields (in each subplot, from bottom to top, $B=0.0 \sim 8.0 \mathrm{~T}$ is increased on steps of $0.2 \mathrm{~T}$ ). (a),(b) for heavy-hole and (c),(d) for light-hole excitons. (b) and (d) are the enlarged versions of (a) and (c). Ring radius $R_{0}=20 \mathrm{~nm}$ and width $W=10 \mathrm{~nm}$. The semiconducting band gap $E_{g}$ is taken to be zero. Note that for the sake of clarity curves have been upshifted with a constant.

Fig.8. The low-lying energy levels of InAs nano-rings as a function of the magnetic field for heavy-hole exciton with different ring widths: (a) $W=8 \mathrm{~nm}$, (b) $14 \mathrm{~nm}$, (c) $20 \mathrm{~nm}$ and (d) $30 \mathrm{~nm}$. Other parameters are the same as in Fig. 5.

Fig.9. Imaginary part of linear optical susceptibility of heavy-hole exciton as a function of frequency for different magnetic fields and different ring widths: (a) $W=8 \mathrm{~nm}$, (b) $14 \mathrm{~nm}$, (c) $20 \mathrm{~nm}$ and (d) $30 \mathrm{~nm}$. 


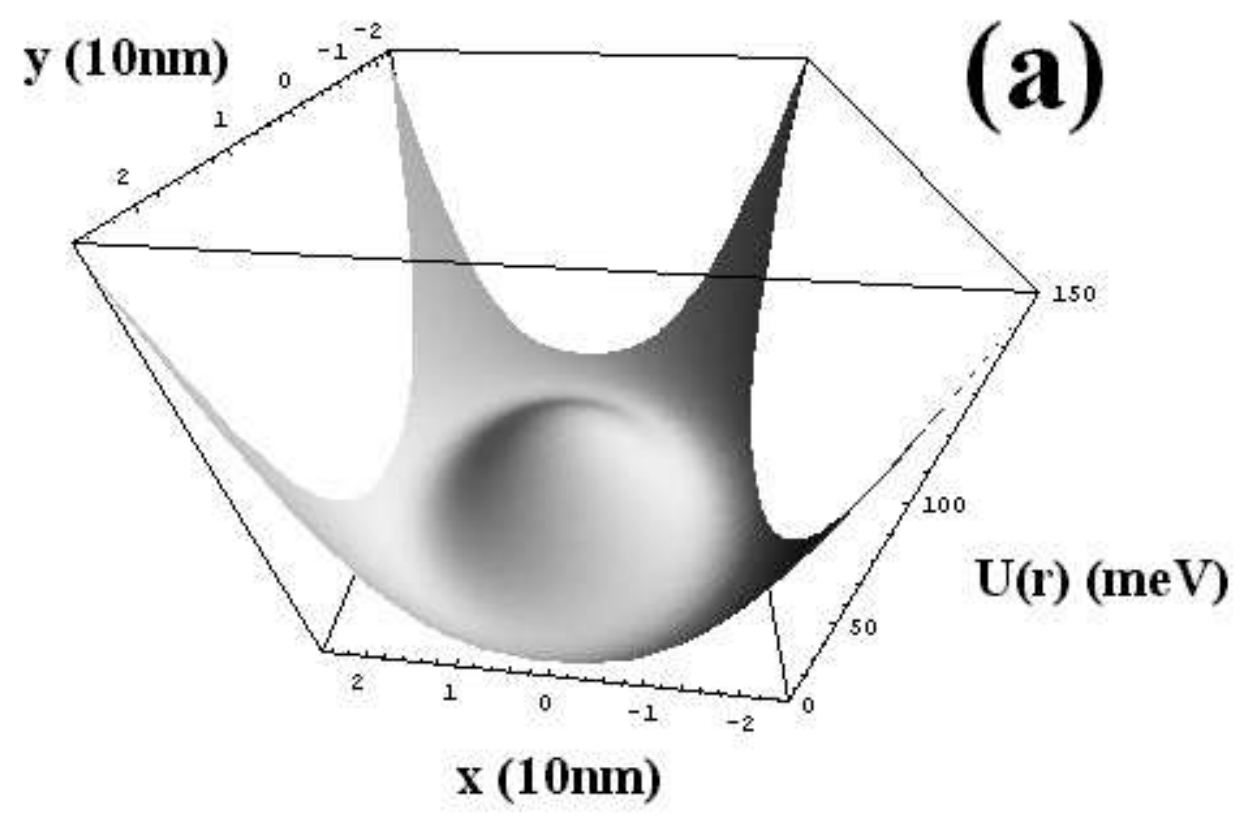

Fig. 1

Aharonov-Bohm effect of excitons in ...... Hui Hu et al.

\section{Fig. 1}

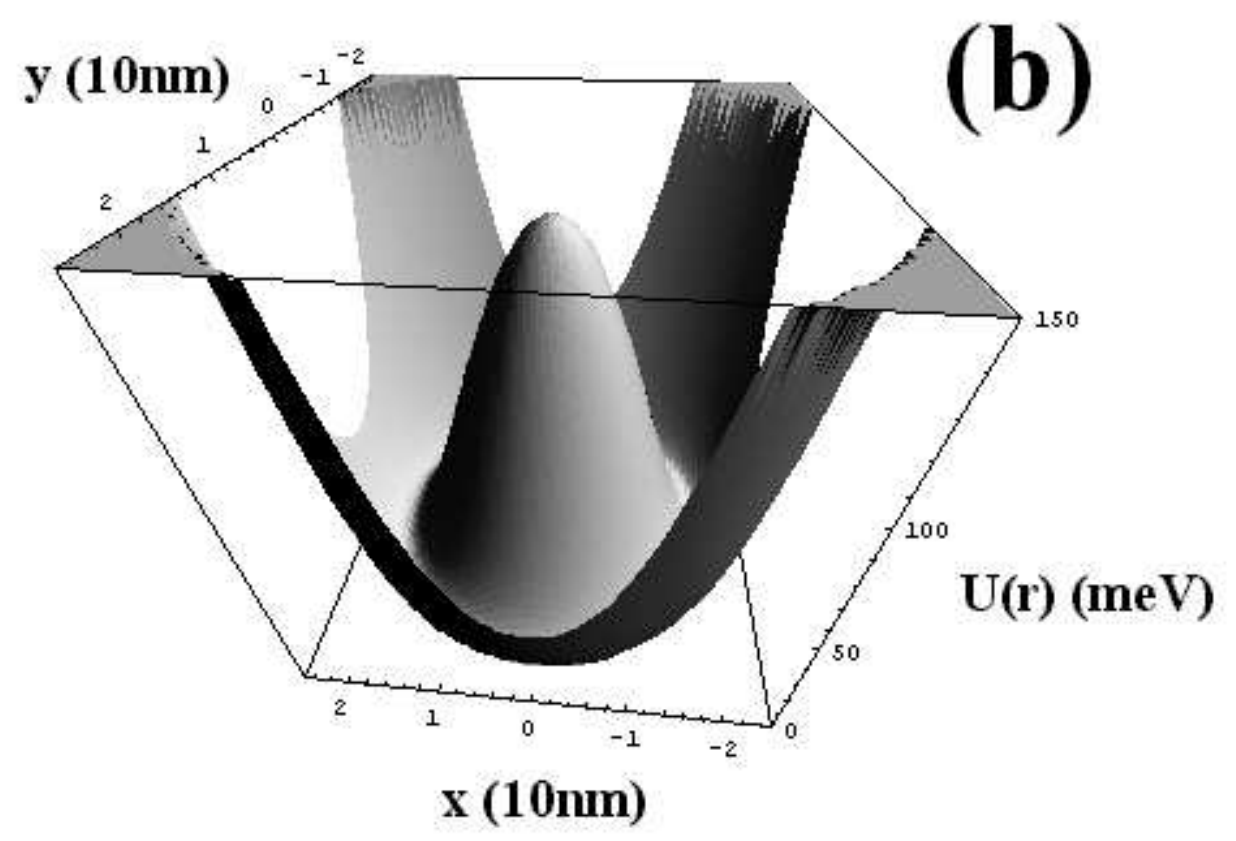




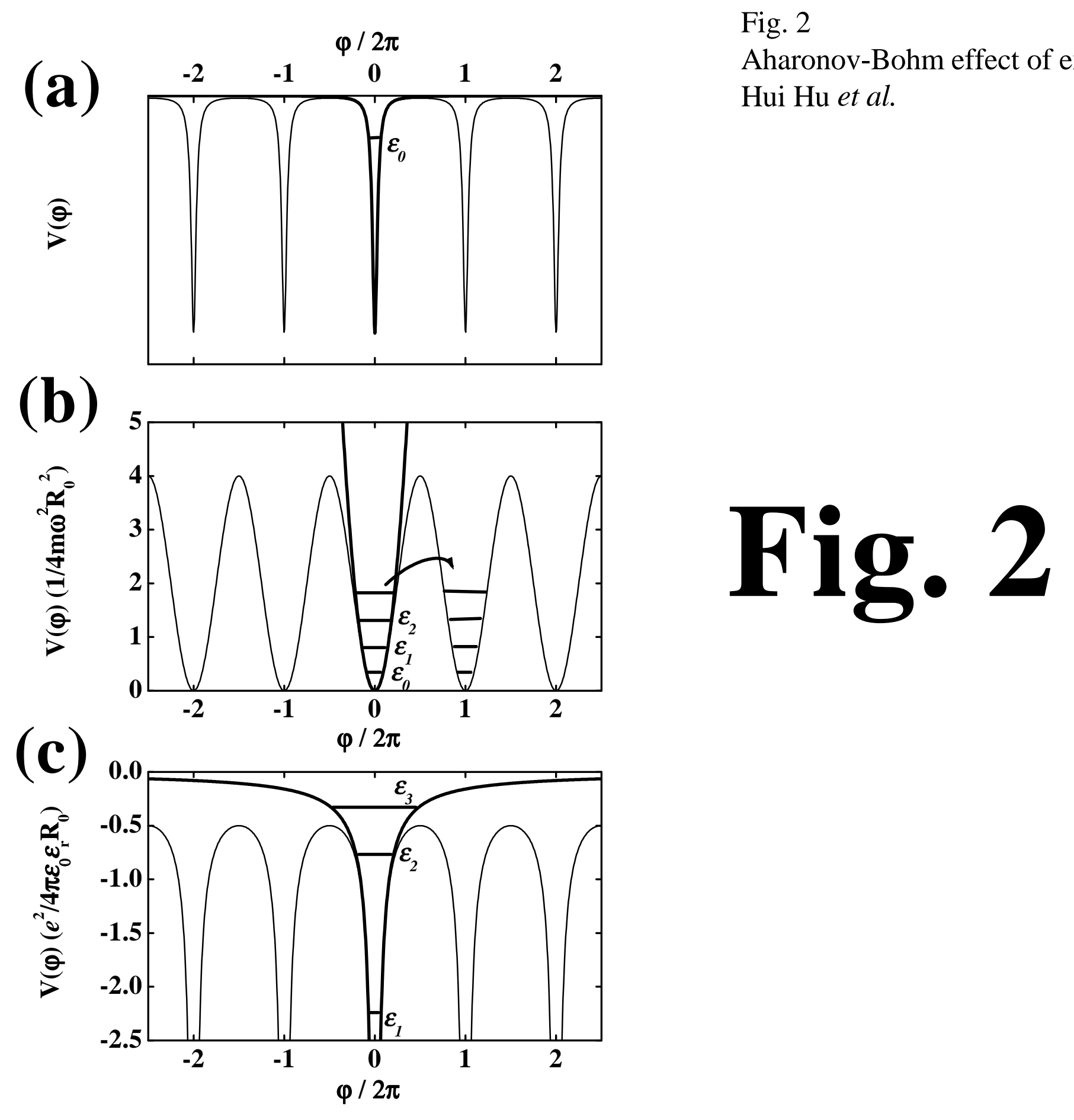



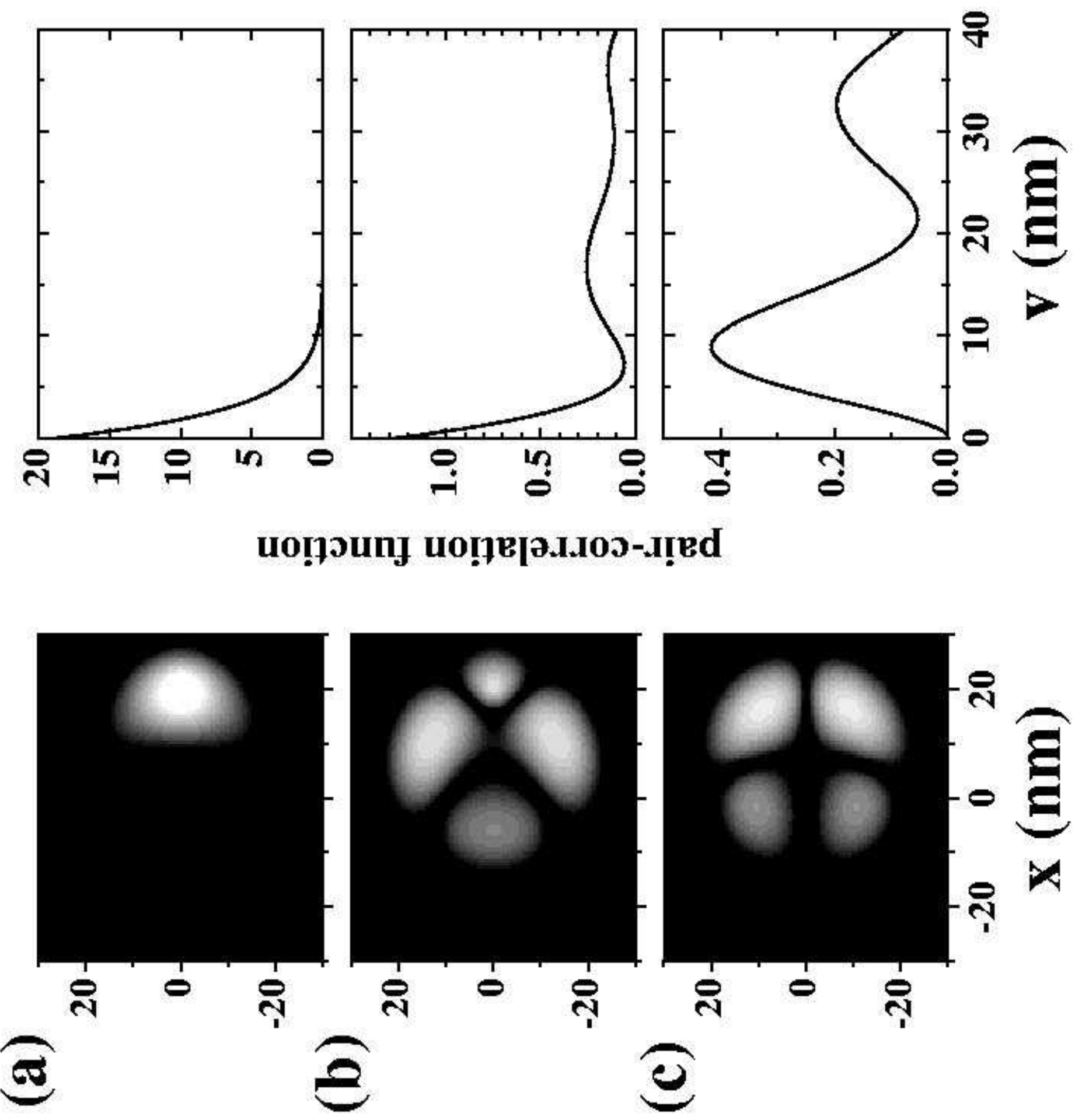

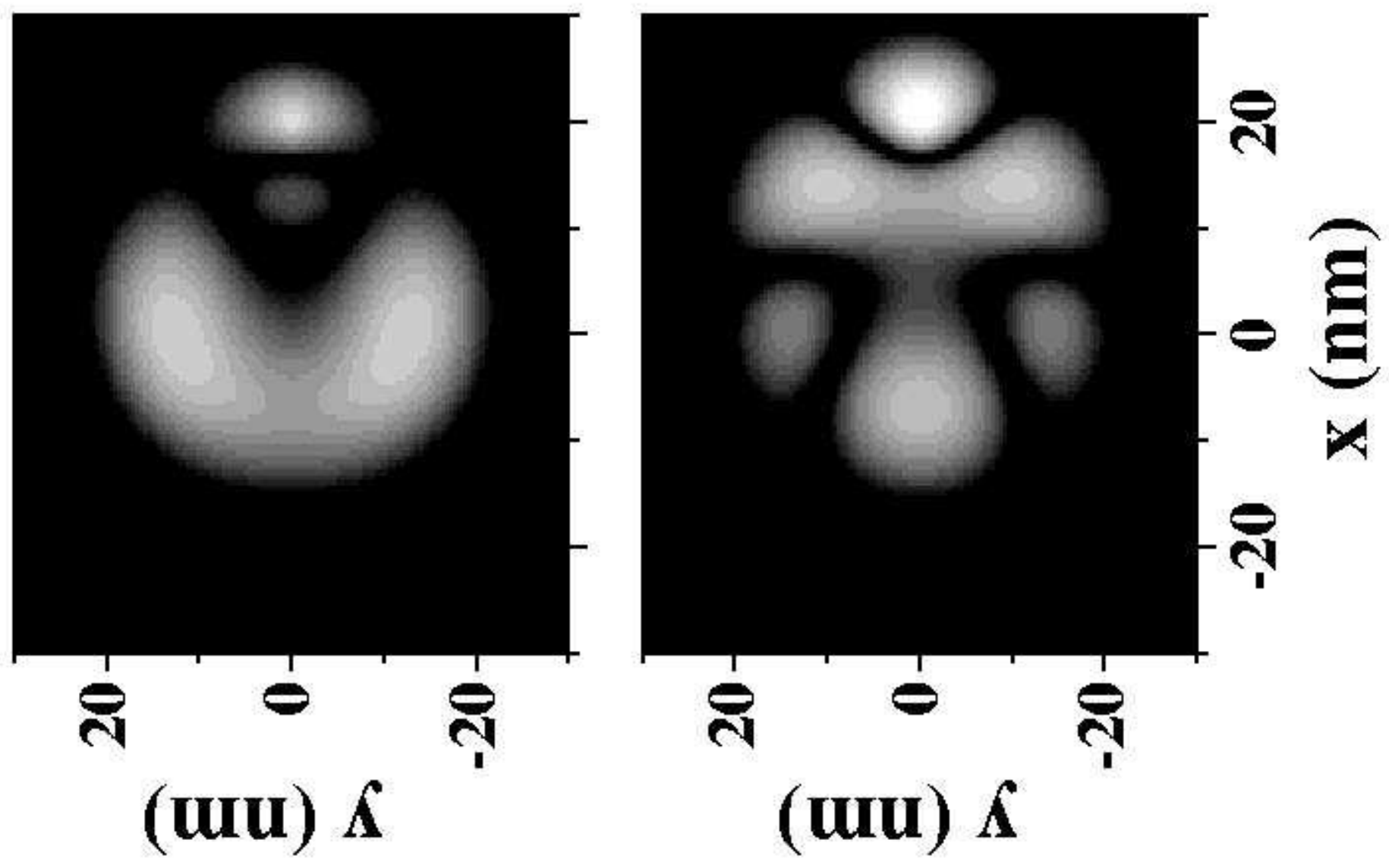
(a)

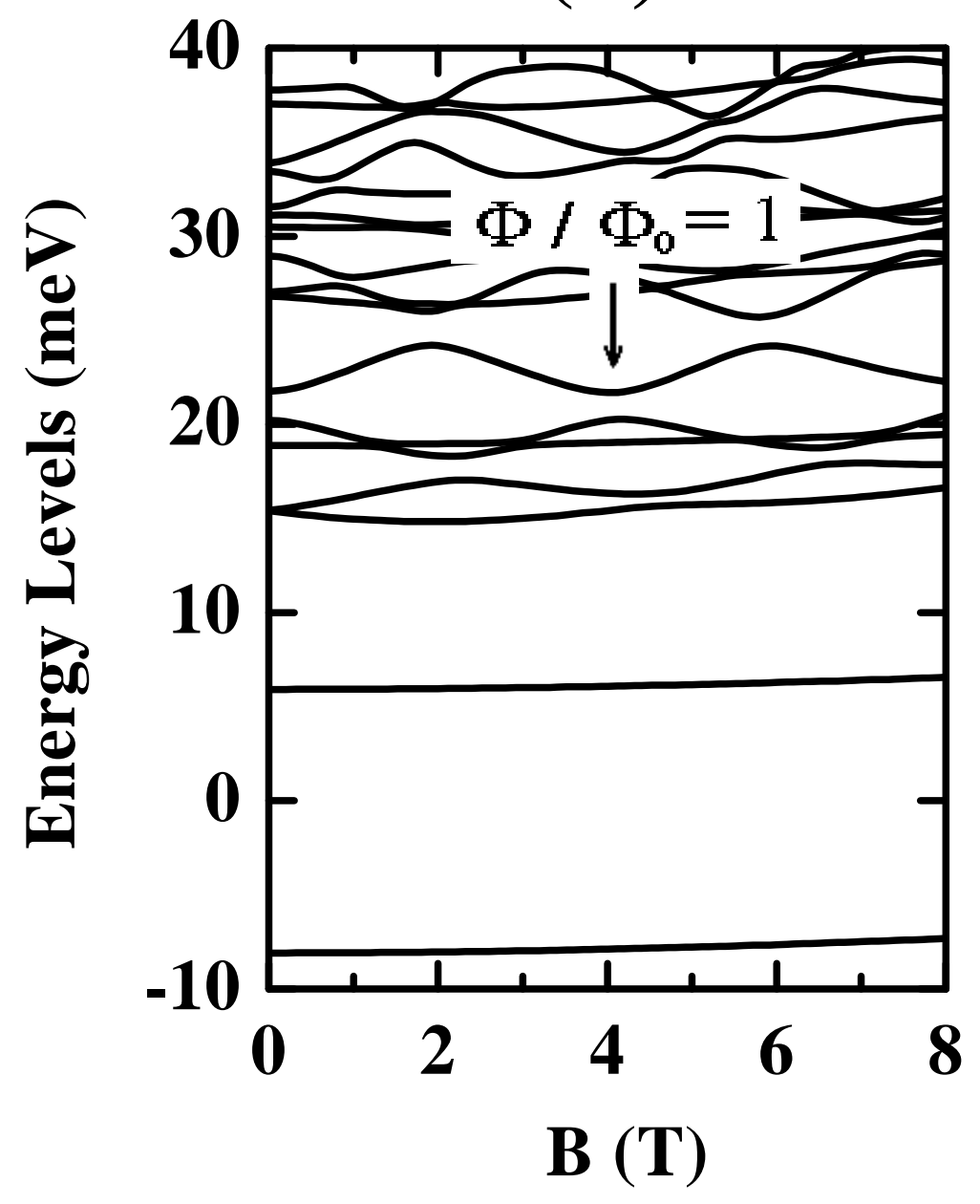

(b)

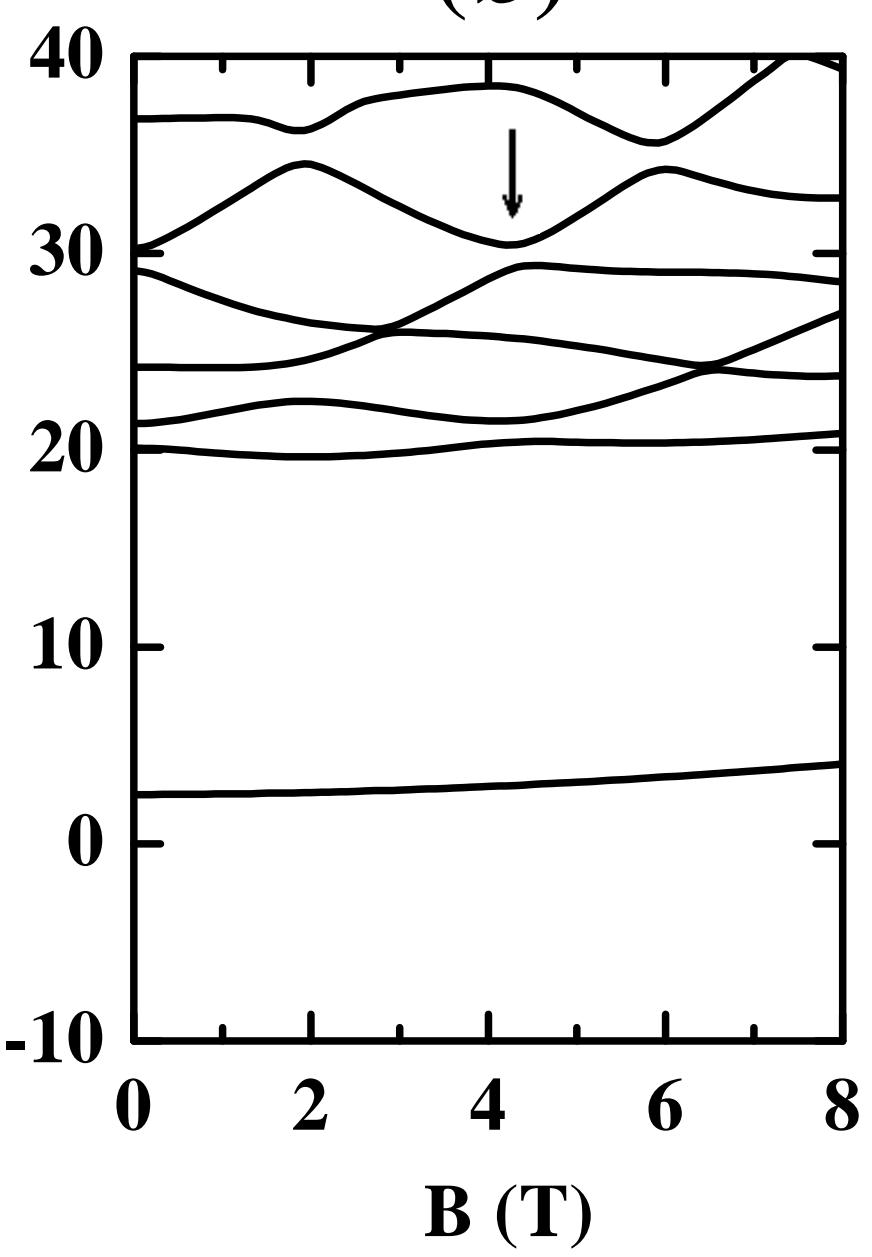

Fig. 5

Aharonov-Bohm effect of excitons in ...... H. Hu et al. 

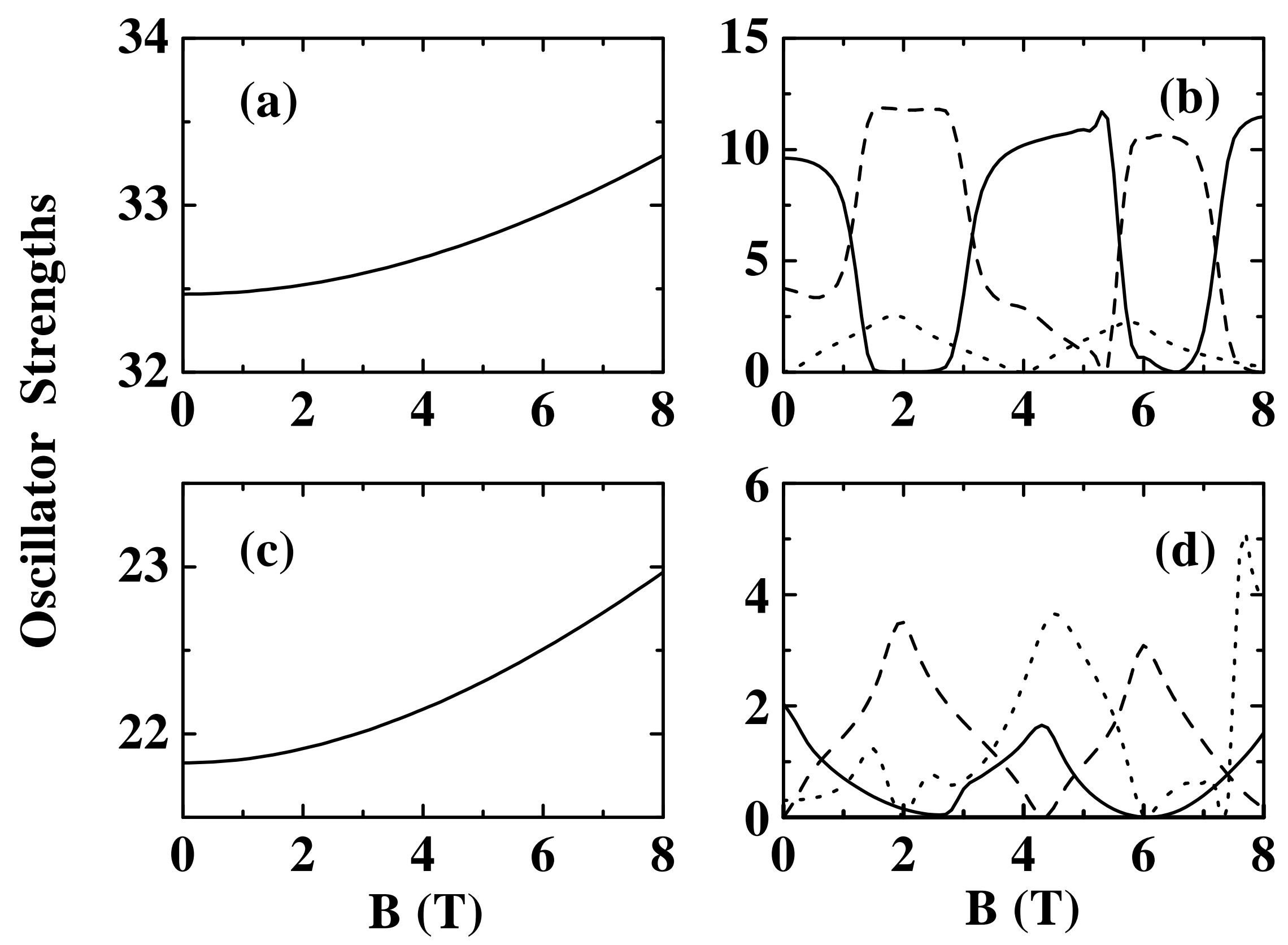

Aharonov-Bohm effect of excitons in ...... 

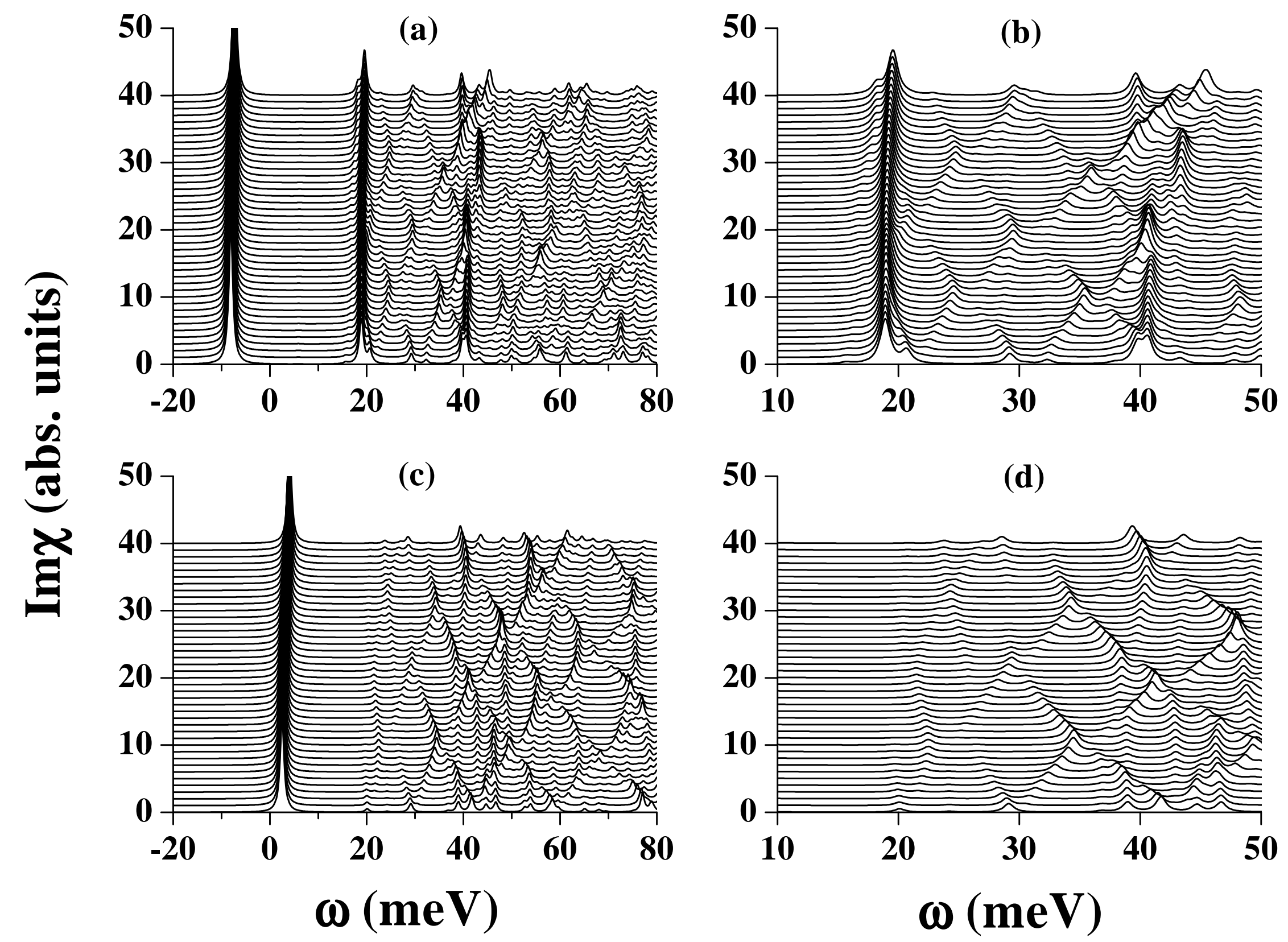

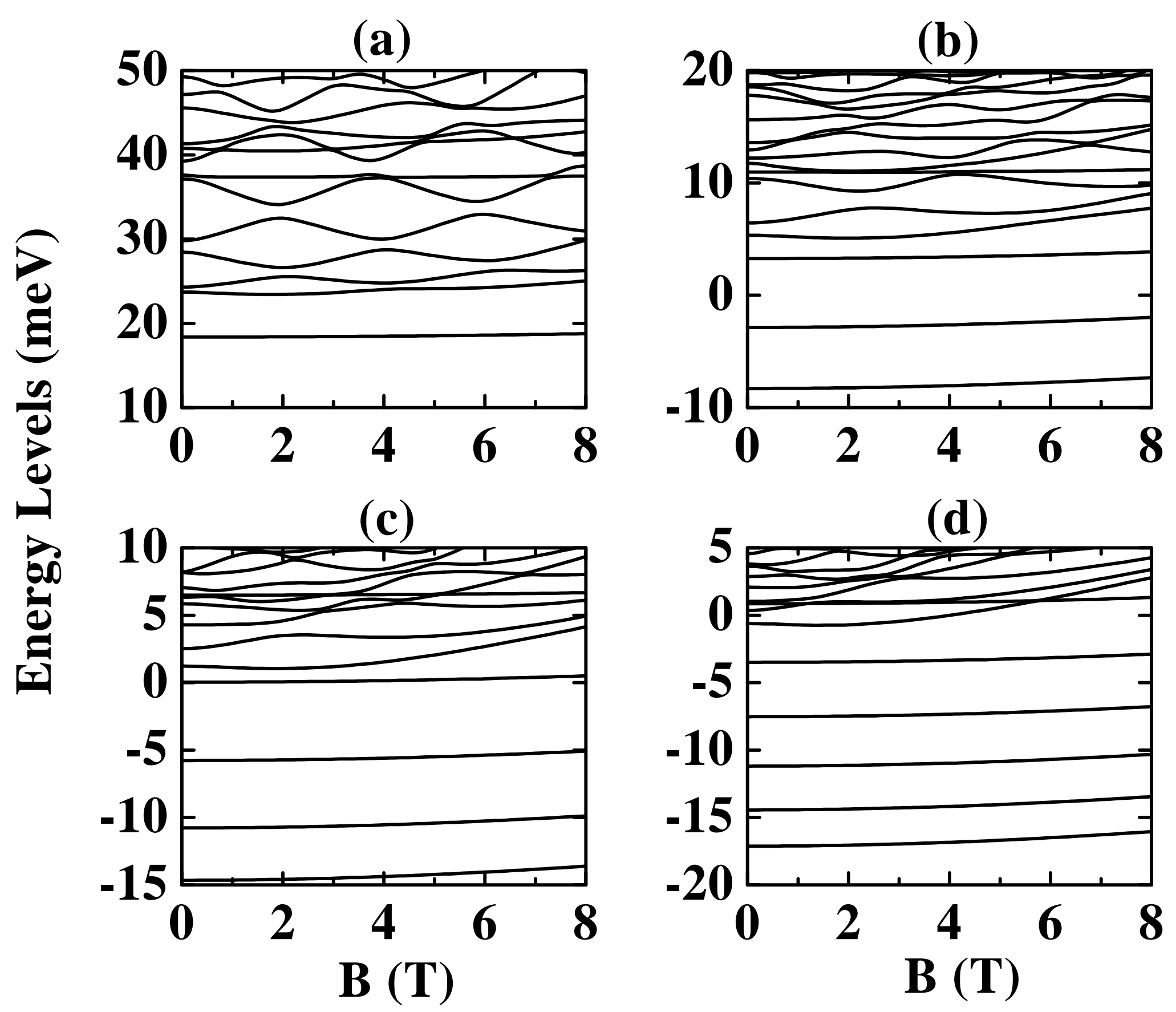

Aharonov-Bohm effect of ......

H. Hu et al. 

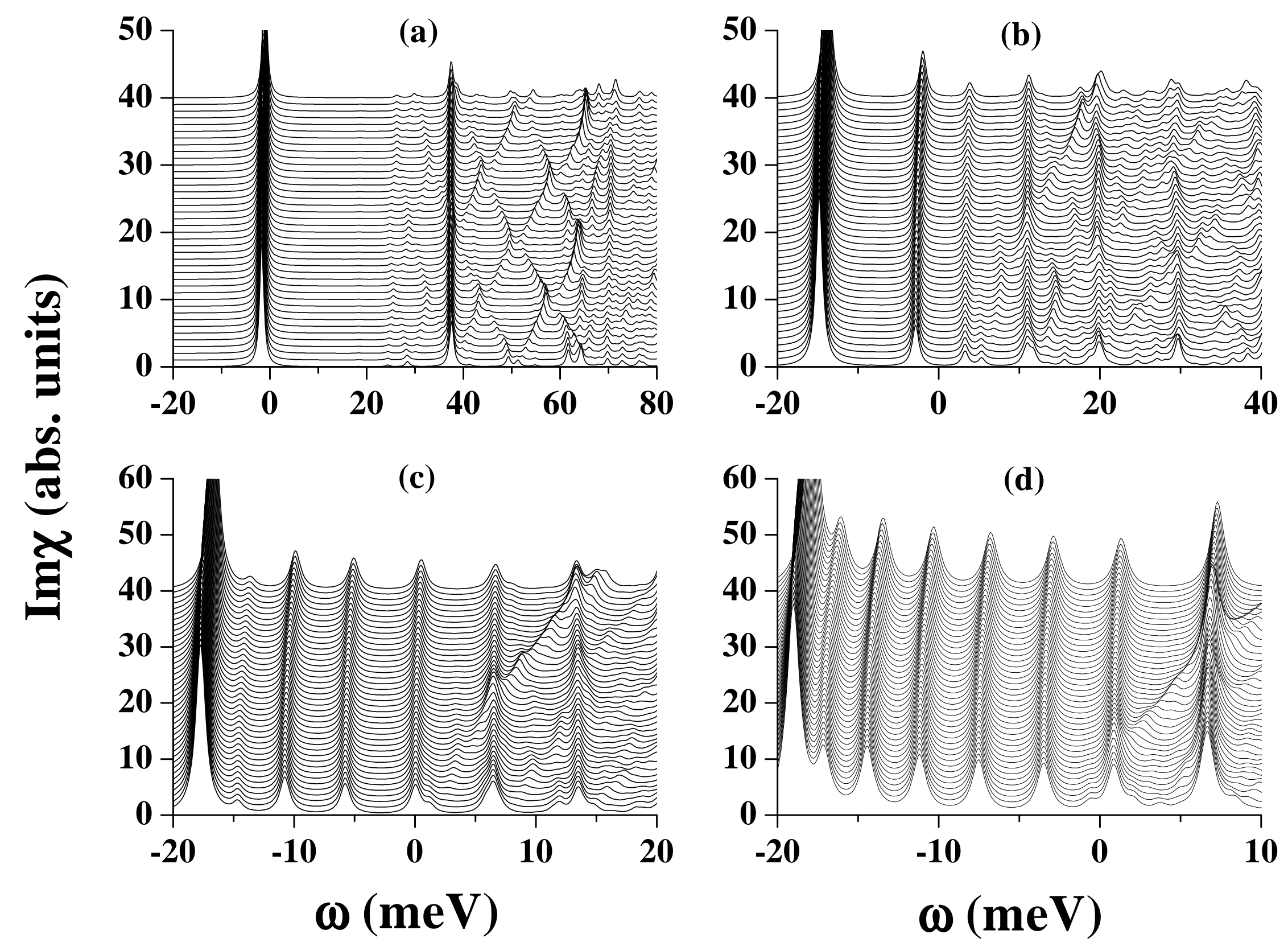

Fig. 9

Aharonov-Bohm effect of excitons in ......

H. Hu et al. 